\title{
Research Article \\ Effect of Nanotube Geometry on the Strength and Dispersion of CNT-Cement Composites
}

\author{
Mohamed O. Mohsen, ${ }^{1}$ Ramzi Taha, ${ }^{1}$ Ala Abu Taqa, ${ }^{1}$ Nasser Al-Nuaimi, ${ }^{1}$ \\ Rashid Abu Al-Rub, ${ }^{2}$ and Khaldoon A. Bani-Hani ${ }^{3}$ \\ ${ }^{1}$ Civil and Architectural Engineering Department, Qatar University, P.O. Box 2713, Doha, Qatar \\ ${ }^{2}$ Mechanical and Materials Engineering Department, Masdar Institute of Science and Technology, P.O. Box 54224, Abu Dhabi, UAE \\ ${ }^{3}$ Civil Engineering Department, Jordan University of Science and Technology, P.O. Box 3030, Irbid 22110, Jordan \\ Correspondence should be addressed to Mohamed O. Mohsen; 200202128@student.qu.edu.qa
}

Received 2 April 2017; Revised 12 May 2017; Accepted 21 June 2017; Published 27 July 2017

Academic Editor: Andrew R. Barron

Copyright (C) 2017 Mohamed O. Mohsen et al. This is an open access article distributed under the Creative Commons Attribution License, which permits unrestricted use, distribution, and reproduction in any medium, provided the original work is properly cited.

\begin{abstract}
This study investigated the effect of multiwalled carbon nanotubes' geometry on the dispersion and strength of cement composites. Mixes with CNTs contents ranging from 0.03 to $0.25 \%$ were prepared and tested using CNTs' diameters of 10-20, 20-30, and $30-50 \mathrm{~nm}$ and lengths of $0.5-2$ and $10-30 \mu \mathrm{m}$. The samples' microstructures were then examined using a scanning electron microscope. After that, Response Surface Methodologies techniques were implemented to determine the strength functions' response to the aspect ratio and weight fraction variables. The results showed that CNTs of smaller diameters and longer lengths resulted in the highest strength values in batches having CNT contents of up to $0.15 \%$. At $0.25 \%$ CNTs, the results showed that those CNTs of shorter lengths attained higher strengths than those of longer ones. SEM images showed better dispersion properties at lower CNTs' contents regardless of their geometry. The analysis also indicated that short CNTs of $0.5-2 \mu \mathrm{m}$ length have better dispersion properties compared to long ones of 10-30 $\mu \mathrm{m}$ length. The RSM model confirmed this finding. Furthermore, the model showed that the CNTs with the lowest aspect ratio of 83 and highest weight fraction of $0.25 \%$ provided the highest strength response among all batches.
\end{abstract}

\section{Introduction}

Carbon nanotubes (CNTs) are molecular-scale shells of graphene placed in hexagonal arrays. They can be comprised of single, double, or multiple shells. CNTs have extraordinary properties such as high strength and thermal and electrical conductivity [1-4]. These properties raised the interest of many concrete and cementitious composites researchers to incorporate the nanofilaments into cement pastes and mortars to develop their properties [5-19]. However, up to date, it was certainly shown that findings reported in the literature were contradictory. Some results confirmed an optimization of the tested composites mechanical properties, while others showed no improvement or, in some cases, there was a reduction in the properties. Most studies related the effects of CNTs' addition on the mechanical properties of cement materials to the different selections of carbon nanofilaments' aspect ratio and weight fraction, which in return will affect the dispersion of the filaments within the solution and the matrix. The unique high aspect ratio (length to diameter ratio) property of CNTs provides a high surface area to volume ratio that allows for additional contact between the tubes and the adjoining hydration products. However, CNTs of very high aspect ratios are harder to disperse than CNTs of low aspect ratios [20]. The effect of CNTs' aspect ratio on the mechanical properties of cement paste was rarely investigated. Konsta-Gdoutos et al. [21] conducted a research on the effects of MWCNTs' content and aspect ratio on both the mechanical properties and fresh paste properties of cement. The study samples were tested on the 3rd, 7th, and 28th days. The CNTs used were long CNTs with an aspect ratio of 1600 and short CNTs with an aspect ratio of 700. The results showed that low quantities of long CNTs between 0.025 and $0.048 \%$ of cement improved the mechanical properties of 
TABLE 1: Experiment design.

\begin{tabular}{|c|c|c|c|c|c|c|c|}
\hline Batch \# & Batch name & $\begin{array}{c}\mathrm{CNT} / \mathrm{cement} \\
\text { weight fraction (\%) }\end{array}$ & Specimen \# & $\begin{array}{l}\text { CNTs diameter } \\
(\mathrm{nm})\end{array}$ & CNTs length $(\mu \mathrm{m})$ & Aspect ratio & $\begin{array}{l}\text { Number of } \\
\text { specimens }\end{array}$ \\
\hline \multicolumn{8}{|c|}{ Effect of diameter test batches } \\
\hline 1 & $0.03(\mathrm{LD})$ & 0.03 & S1-S18 & $10-20$ & $10-30$ & 1333 & 18 \\
\hline 2 & $0.03(\mathrm{MD})$ & 0.03 & S19-S36 & $20-30$ & $10-30$ & 800 & 18 \\
\hline 3 & $0.03(\mathrm{HD})$ & 0.03 & S37-S54 & $30-50$ & $10-30$ & 375 & 18 \\
\hline 4 & $0.08(\mathrm{LD})$ & 0.08 & S55-S72 & $10-20$ & $10-30$ & 1333 & 18 \\
\hline 5 & $0.08(\mathrm{MD})$ & 0.08 & S73-S90 & $20-30$ & $10-30$ & 800 & 18 \\
\hline 6 & $0.08(\mathrm{HD})$ & 0.08 & S91-S108 & $30-50$ & $10-30$ & 375 & 18 \\
\hline 7 & $0.15(\mathrm{LD})$ & 0.15 & S109-S126 & $10-20$ & $10-30$ & 1333 & 18 \\
\hline 8 & $0.15(\mathrm{MD})$ & 0.15 & S127-S144 & $20-30$ & $10-30$ & 800 & 18 \\
\hline 9 & $0.15(\mathrm{HD})$ & 0.15 & S145-S162 & $30-50$ & $10-30$ & 375 & 18 \\
\hline 10 & $0.25(\mathrm{LD})$ & 0.25 & S163-S180 & $10-20$ & $10-30$ & 1333 & 18 \\
\hline 11 & $0.25(\mathrm{MD})$ & 0.25 & S181-S198 & $20-30$ & $10-30$ & 800 & 18 \\
\hline 12 & $0.25(\mathrm{HD})$ & 0.25 & S199-S216 & $30-50$ & $10-30$ & 375 & 18 \\
\hline \multicolumn{8}{|c|}{ Additional batches to compare the effect of length } \\
\hline 13 & $0.03(\mathrm{LL})$ & 0.03 & S217-S234 & $10-30$ & $0.5-2$ & 83 & 18 \\
\hline 14 & $0.08(\mathrm{LL})$ & 0.08 & S235-S252 & $10-30$ & $0.5-2$ & 83 & 18 \\
\hline 15 & $0.15(\mathrm{LL})$ & 0.15 & S253-S270 & $10-30$ & $0.5-2$ & 83 & 18 \\
\hline 16 & $0.25(\mathrm{LL})$ & 0.25 & S271-S288 & $10-30$ & $0.5-2$ & 83 & 18 \\
\hline 17 & Cement (control) & 0 & S289-S306 & - & - & - & 18 \\
\hline
\end{tabular}

cement paste. On the other hand, the results illustrated that higher quantities of short CNTs (about $0.08 \%$ of cement weight) are needed to reach the same level. The strength gain of all CNTs' batches showed a similar incremental behavior to the plain cement paste batches between the testing dates. Abu Al-Rub et al. [8] studied the effect of several weight fractions of long MWCNTs with aspect ratios of 1,250-3,750 and short MWCNTs with an aspect ratio of about 150 on the mechanical properties of cementitious composites. The samples were tested on the 7th, 14th, and 28th days. The long MWCNTs had a diameter of $9.5 \mathrm{~nm}$ and a length of $1.5 \mu \mathrm{m}$, whereas the short ones had diameters less than $8 \mathrm{~nm}$ and lengths between 10 and $30 \mu \mathrm{m}$. The obtained results showed that the addition of $0.2 \%$ short MWCNTs and $0.1 \%$ long MWCNTs increased the flexural strength by 269 and 65\%, respectively, compared to the control cement paste mix. Furthermore, the results showed a reduction in the strength between the 14th and the 28th days of few batches. The authors related the purpose of this reduction to the nonuniform dispersion, weak bonding, formation of weak hydration products, formation of expansive ettringite, and leaching of calcium hydroxide. Up to date, no research was performed on studying the role of CNTs' diameters of various weight fractions on the strength and dispersion of cement and CNTs composites. The aspect ratio changes if the tube length and/or tube diameter changes.

Therefore, the purpose of this study is to investigate the effect of MWCNTs' diameter and length on the dispersion and flexural and compressive strength gain of cement paste. The microstructures of the samples were studied using scanning electron microscope (SEM) to investigate the factors strength results.

\section{Testing Methodology}

Two CNTs' geometrical variables, which are their diameter and length, were tested with several CNTs-to-cement weight fractions. Table 1 shows the experiment design indicating the prepared batches, geometrical parameters, and CNTs' weight fraction. The testing methodology commenced by preparing the samples, followed by measuring their compressive and flexural strengths using a three-point bending test and a cubic uniaxial test, respectively. Then, the fractured samples microstructures were examined by capturing SEM images from each batch. After that, CNTs' dispersion within the cement hydration products was then qualitatively evaluated. Then, an RSM model was created to determine the flexural and compressive strength functions' response to the aspect ratio and weight fraction variables. Finally, the relationships between CNTs' geometrical parameters, strength, and dispersion were reported.

2.1. Materials and Equipment. Portland cement CEM I $42.5 \mathrm{R}$ was used throughout this experiment. To prevent any contact with air moistures, it was stored in sealed bags during the test period. Nontreated multiwalled carbon nanotubes differentiated by lengths and diameters were used. They were supplied by US Research Nanomaterials, Inc. The surfactant used to disperse the nanofilaments within the aqueous solution was a polycarboxylate superplasticizer of a commercial name GRACE ADVA 575. The equipment employed in performing the study experiment is comprised of an ultrasonic wave sonicator, a cement paste mixer, a strength testing machine provided by Controls Inc., a scanning electron microscope, 


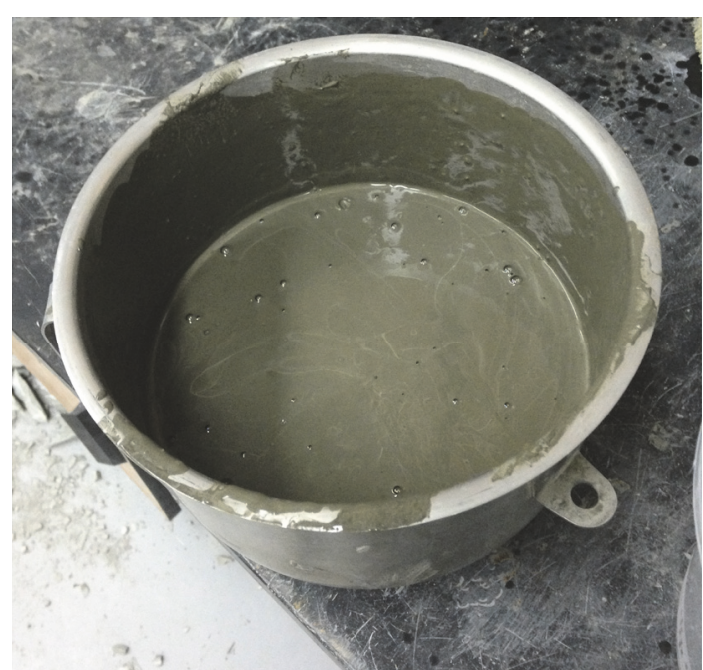

(a)

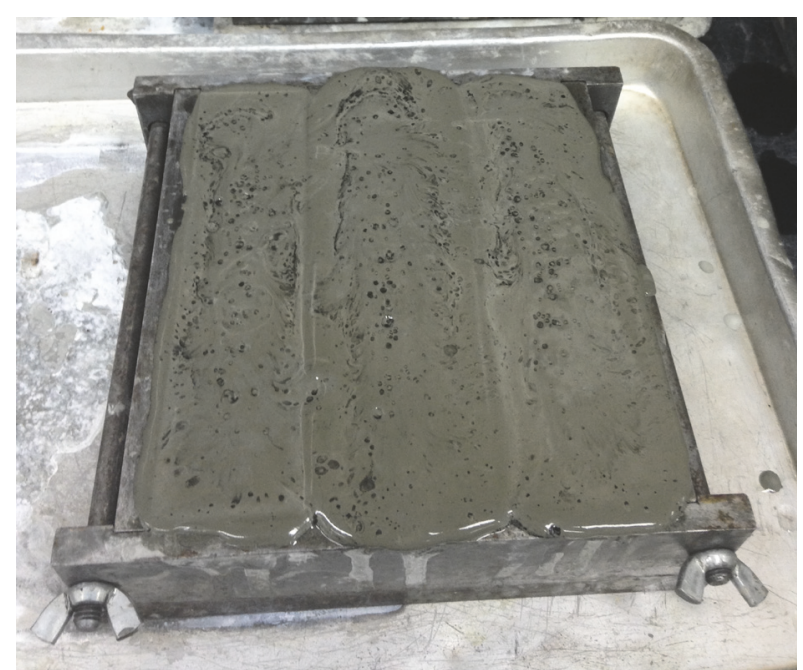

(b)

FIGURE 1: (a) Mixing of cement composite batch. (b) Sample of casted specimens including CNTs.

$40 \times 40 \times 160 \mathrm{~mm}$ steel molds for flexural testing, and $50 \times$ $50 \times 50 \mathrm{~mm}$ steel molds for compression testing.

\subsection{Mixing Method. Batches mixing started by measur-} ing cement, water, surfactant, and CNTs weights. In each batch, the cement and water quantities used were 7,000 and 2,800 gm, respectively (considering a water-to-cement ratio of 0.4 ). The surfactant weight was set at $4: 1$ of the chosen CNTs' weight fraction. The temperature of the water was set at $23^{\circ} \mathrm{C}$. First, CNTs were mixed with water and the surfactant. Then, the sonication process was carried out for $30 \mathrm{~min}$ at amplitude of $70 \%$. The solution temperature was kept less than $30^{\circ} \mathrm{C}$ to prevent any water evaporation. After the completion of the sonication process, the solution was kept to cool down until its temperature reached $23^{\circ} \mathrm{C}$. Then, the water/CNT solution was mixed with cement. The procedure of mixing was comprised of first installing the water/CNT solution in the mixer and then adding the cement quantities in five portions, while the mixer is operating at a slow speed of $140 \mathrm{rpm}$. This process lasted about $10 \mathrm{~min}$. After adding the entire cement amount (Figure 1(a)), the mixing carried on for another $5 \mathrm{~min}$ at a medium speed of $285 \mathrm{rpm}$. The mix was then poured into the molds and compacted as per ASTM C348 standard (Figure 1(b)). Finally, the samples were demolded after 24 hours and installed in a curing tank.

2.3. Flexural and Compressive Strength Tests. The three-point flexural test of the samples (Figure 2(a)) was conducted according to ASTM C348 standard test method for flexural strength of hydraulic-cement mortars. The loading rate was set at $41 \mathrm{~N} / \mathrm{sec}$. On the other hand, the compression test (Figure 2(b)) was conducted according to ASTM C109 standard test method for compressive strength of hydraulic cement mortars. The loading rate used was set at 1,400 N/sec.

2.4. Microstructural Analysis. The microstructural analysis of the fractures samples using SEM was performed to understand the effect of the CNTs' diameters and lengths on the flexural and compressive strengths. The samples were first dried using a vacuum and then coated using gold palladium to dissipate excess charges and to enhance images resolution. After that, low-energy Secondary Electron (SE) imaging option was used to capture images with scales between 1 and $5 \mu \mathrm{m}$.

2.5. RSM Analysis. The influence of CNTs' aspect ratio and weight fraction variables on the 28th day flexural and compressive strength factors' response was investigated using Response Surface Methodologies (RSM) techniques. Using JMP software, the Central Composite Design (CCD) method was used to produce surface plots, contour profiles, and maximized desirability functions between the study variables. The methodology followed to perform this analysis was as follows:

(1) The CNTs' aspect ratio and weight fractions variables were coded in the $(-1,1)$ interval (Table 2$)$.

(2) The factors were modeled by fitting a second-order polynomial equation model.

Second-order polynomial equations were used to express the flexural and compressive strength as functions of independent variables:

$$
\begin{aligned}
& \mathrm{FS}=a_{0}+a_{1} x_{1}+a_{2} x_{2}+a_{11} x_{1}{ }^{2}+a_{22} x^{2}+a_{12} x_{1} x_{2} \\
& \mathrm{CS}=b_{0}+b_{1} x_{1}+b_{2} x_{2}+b_{11} x_{1}{ }^{2}+b_{22} x^{2}+b_{12} x_{1} x_{2},
\end{aligned}
$$

where FS is flexural strength, (MPa), CS is compressive strength, (MPa), $x_{1}$ is aspect ratio, $x_{2}$ is CNTs-to-cement weight fraction, (\%), and $a_{0}, a_{1}, a_{2}, a_{11}, a_{22}, a_{12}, b_{0}, b_{1}, b_{2}, b_{11}$, $b_{22}$, and $b_{12}$ are the response surface coefficients.

(3) The response surface coefficients were determined by using a CCD design type with 2 center points.

(4) The prediction models, $R$-squared and $P$ values, were recorded.

(5) The response surface and contour lines were plotted. 


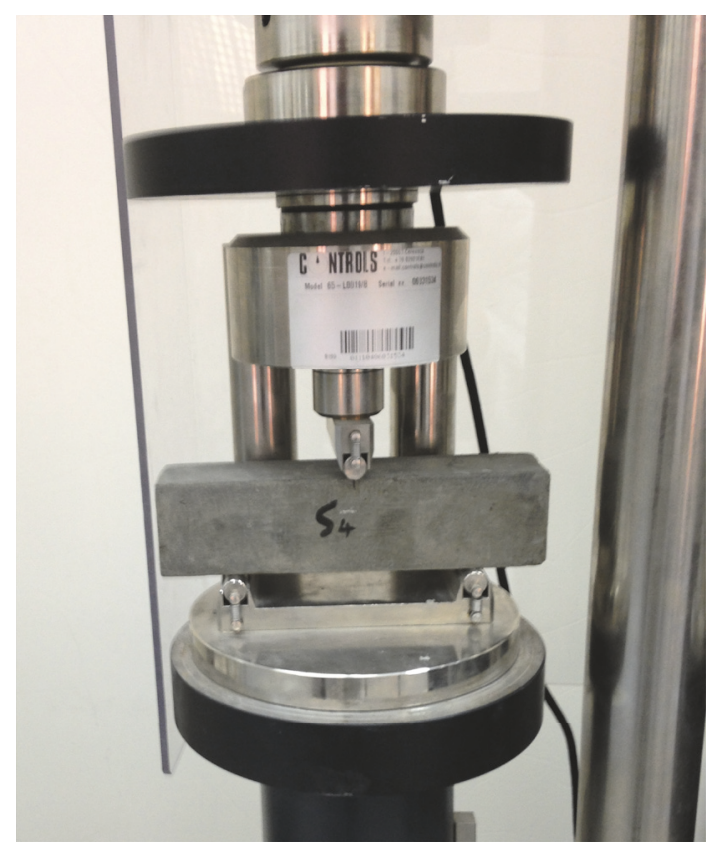

(a)

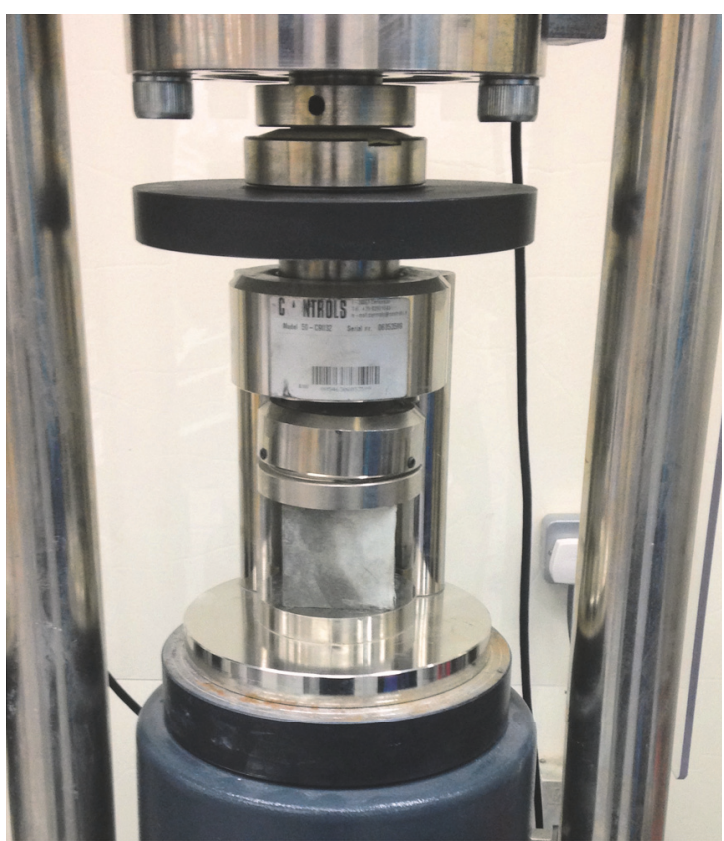

(b)

FIGURE 2: (a) Flexural strength test and (b) compressive strength test.

TABLE 2: Coding of aspect ratio and weight fractions variables for RSM analysis.

\begin{tabular}{lcccc}
\hline Batch & Aspect ratio & Coded aspect ratio & Weight fraction (wt.\%) & Coded weight fraction \\
\hline $0.03 \mathrm{LL}$ & 83 & -1 & 0.03 & -1 \\
$0.08 \mathrm{LL}$ & 83 & -1 & 0.08 & -0.545 \\
$0.15 \mathrm{LL}$ & 83 & -1 & 0.15 & 0.09 \\
$0.25 \mathrm{LL}$ & 83 & -1 & 0.25 & 1 \\
$0.03 \mathrm{HD}$ & 375 & -0.53 & 0.03 & -1 \\
$0.08 \mathrm{HD}$ & 375 & -0.53 & 0.08 & -0.545 \\
$0.15 \mathrm{HD}$ & 375 & -0.53 & 0.15 & 0.09 \\
$0.25 \mathrm{HD}$ & 375 & -0.53 & 0.25 & 1 \\
$0.03 \mathrm{MD}$ & 800 & 0.147 & 0.03 & -1 \\
$0.08 \mathrm{MD}$ & 800 & 0.147 & 0.08 & -0.545 \\
$0.15 \mathrm{MD}$ & 800 & 0.147 & 0.15 & 0.09 \\
$0.25 \mathrm{MD}$ & 800 & 0.147 & 0.25 & 1 \\
$0.03 \mathrm{LD}$ & 1,333 & 1 & 0.03 & -1 \\
$0.08 \mathrm{LD}$ & 1,333 & 1 & 0.08 & -0.545 \\
$0.15 \mathrm{LD}$ & 1,333 & 1 & 0.15 & 0.09 \\
$0.25 \mathrm{LD}$ & 1,333 & 1 & 0.25 & 1 \\
\hline
\end{tabular}

(6) The variables resulting in maximum responses were determined by optimizing the desirability function.

\section{Results and Discussion}

\subsection{Flexural and Compressive Strength Testing}

3.1.1. Effect of CNTs' Diameter. Figure 3 shows the flexural strength's results of the diameter test samples showing the standard deviation in every batch. The flexural strength's results of the batches incorporating CNTs were relatively high. This was also seen in some previous literature studies $[8,9,22]$. In general, the strength results indicated that CNTs with low diameters and high aspect ratios achieved the highest flexural strengths regardless of the CNTs' content. This may be related to the larger contact area between the CNTs' surfaces and the surrounding cement matrix compared to the other types of medium and large diameters.

On the 3rd and 7th days, the flexural strengths of the batches containing low CNTs' content of 0.03 and $0.08 \%$ were lower than plain cement batch strength, regardless of the CNTs' diameter. On the other hand, the flexural strengths 


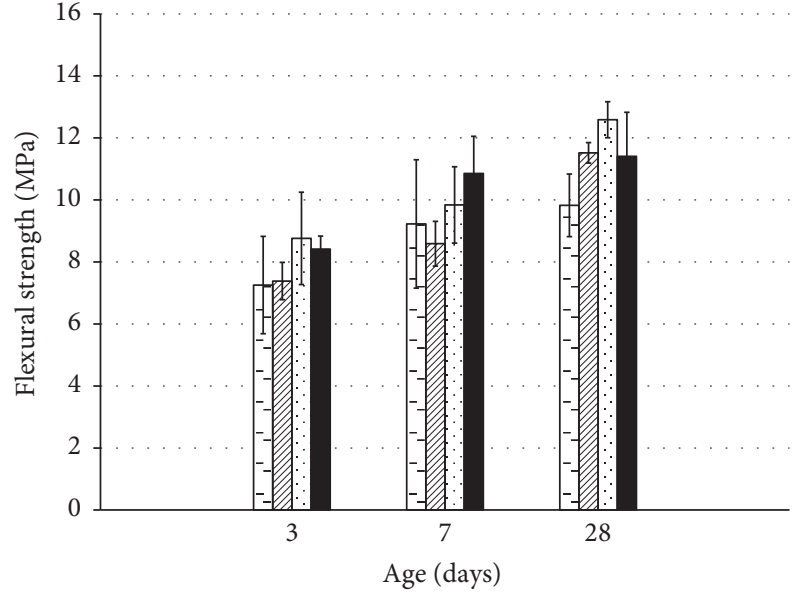

$0.03(\mathrm{HD})$

$0.03(\mathrm{MD})$

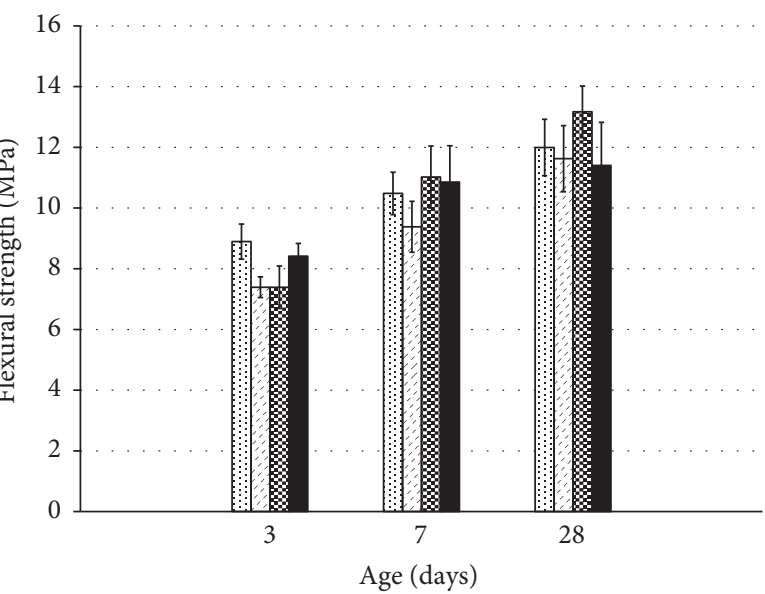

$0.15(\mathrm{HD})$

$0.15(\mathrm{MD})$

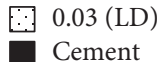

ख $0.15(\mathrm{LD})$

Cement

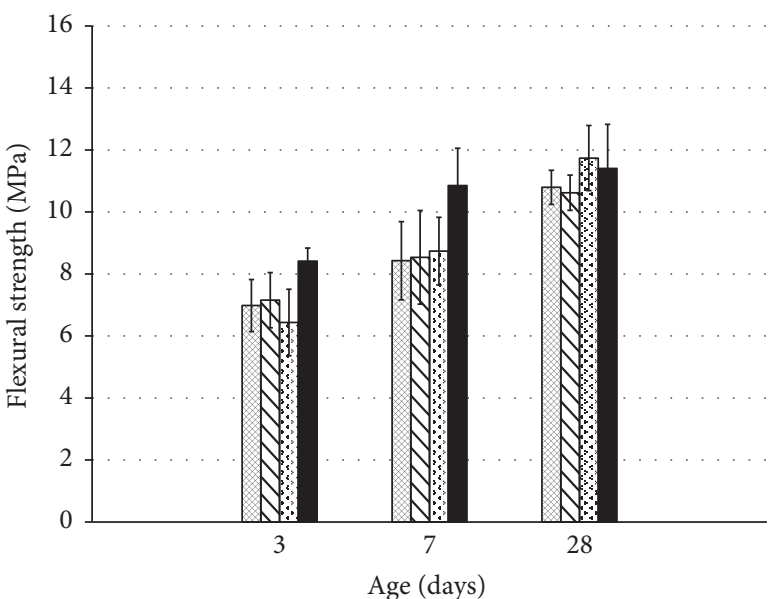

$0.08(\mathrm{HD})$

$0.08(\mathrm{MD})$

0.08 (LD)

Cement (b)

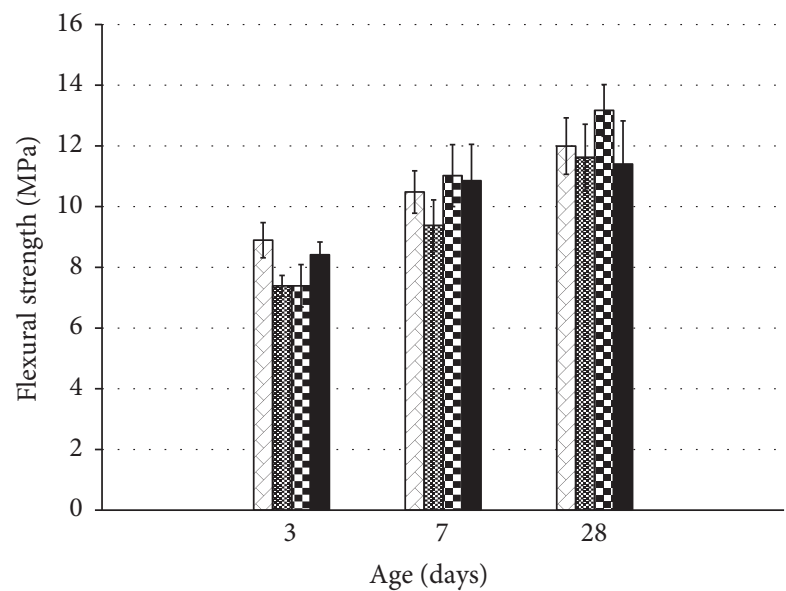

$0.25(\mathrm{HD})$

$0.25(\mathrm{MD})$

(c)

Age (days)

0.25 (LD)

Cement

(d)

FIGURE 3: CNTs' diameter test flexural strength results. (a) $0.03 \%$, (b) $0.08 \%$, (c) $0.15 \%$, and (d) $0.25 \%$ CNTs.

of the batches with higher CNTs' content of 0.15 and $0.25 \%$ were comparable to the plain cement batch's strength. This finding highlights the role of CNTs in the hydration process. On the 28th day and for batches prepared at $0.03 \%$ CNTs, the flexural strength increased by 14 and $22 \%$ at the medium and low CNTs' diameters of 20-30 and 10-20 nm, respectively (Figure 3(a)). However, for batches prepared with 0.08, 0.15, and $0.25 \%$, the flexural strength leveled off between high and medium CNTs' diameters of 30-50 and 20-30 nm and then increased by $10 \%$ at low CNTs' diameters of $10-20 \mathrm{~nm}$ (Figures 3(b), 3(c), and 3(d)). Furthermore, Figures 3(c) and 3(d) showed clear increments in the flexural strength for both 0.15 and $0.25 \%$ CNTs that occurred when using low CNTs' diameters of $10-20 \mathrm{~nm}$ compared to the results obtained for the plain cement. For the $0.15 \%$ CNT (Figure 3(c)), an increment of about $16 \%$ in the flexural strength was obtained.
A comparable behavior was also observed for the $0.25 \%$ CNT batches (Figure 3(d)), where the batch mixed with a low CNTs' diameter of 10-20 nm displayed a flexural strength close to $20 \%$ higher than plain cement.

Figure 4 shows the compressive strength results of the diameter effect test batches, including the standard deviation for each batch. Unlike the flexural strength results, low standard deviations in the compressive strength results were observed in the same batches. Furthermore, the results demonstrated that CNTs' diameters did not have a significant impact on the compressive strength. On the 3rd and 7th days, the compressive strengths of all batches containing CNTs were quite lower than plain cement batch's strength, regardless of the CNTs' diameter and weight fraction. On the 28th day, batches prepared using $0.03 \%$ CNTs showed similar strength results to the plain cement batch. However, for 

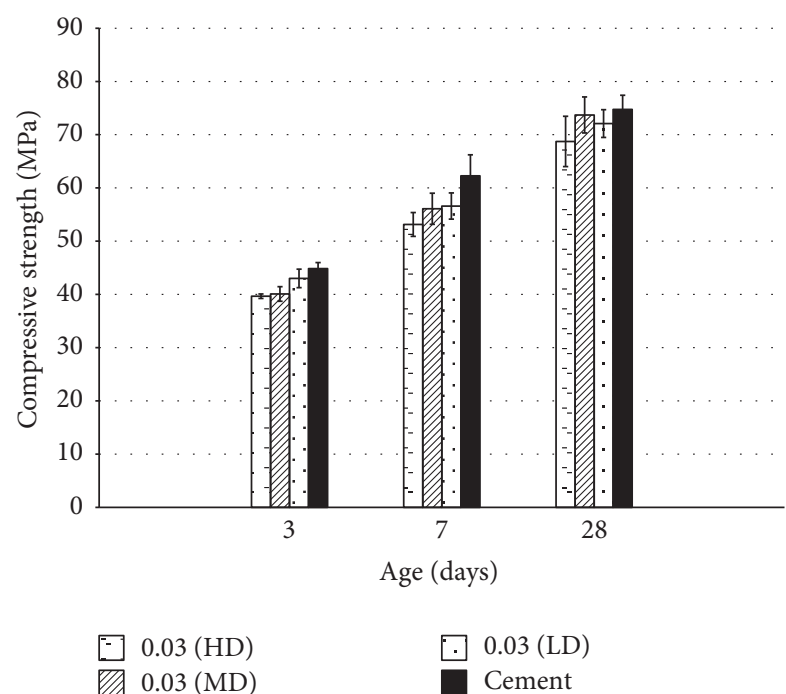

(a)

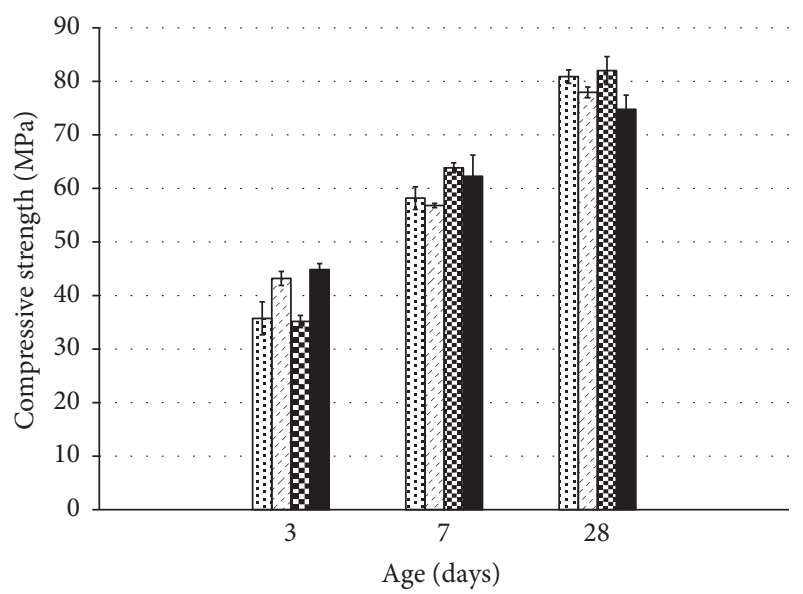

$0.15(\mathrm{HD})$
$0.15(\mathrm{MD})$

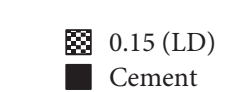

(c)

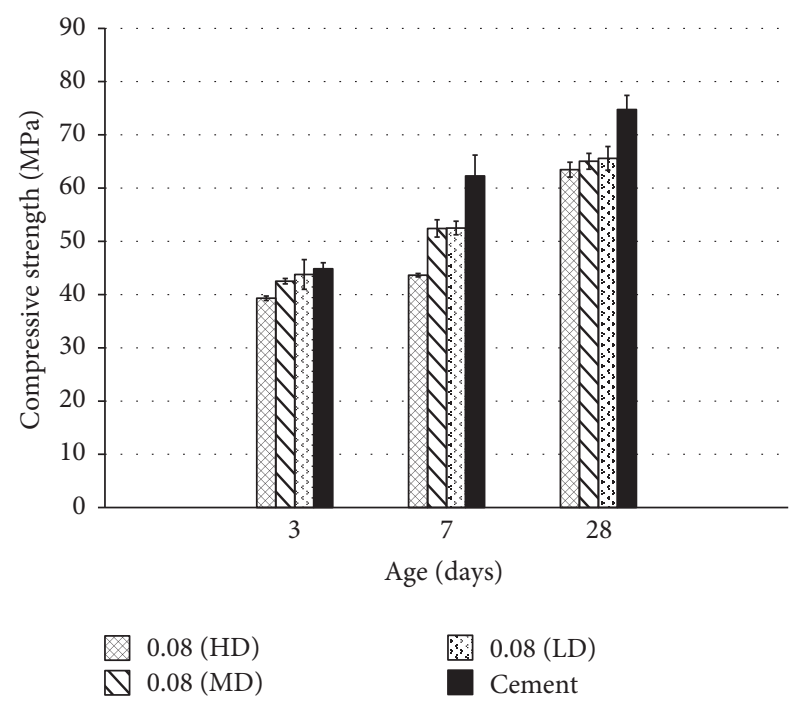

(b)

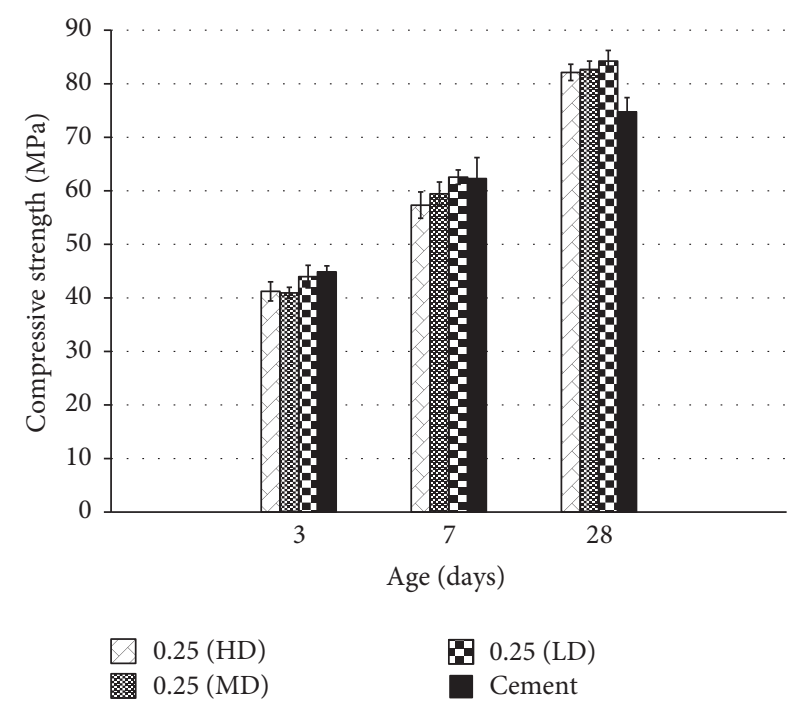

(d)

Figure 4: CNTs' diameter test compressive strength results. (a) $0.03 \%$, (b) $0.08 \%$, (c) $0.15 \%$, and (d) $0.25 \%$ CNTs.

batches prepared using $0.08 \%$ CNTs, the strength data were relatively lower than the plain cement batches. For batches of 0.15 and $0.25 \%$ CNTs, the compressive strength increased by $10 \%$ regardless of the CNTs' diameter type.

3.1.2. Effect of CNTs' Length. Figure 5 shows the effect of CNTs' length on the flexural strength of the cementitious composites. On the 3rd and 7th days, the flexural strengths of the batches containing low CNTs' content of 0.03 and $0.08 \%$ were lower than plain cement batch's strength and the strengths of the batches with higher CNTs' content of 0.15 and $0.25 \%$ were higher than or comparable to the plain cement batch's strength regardless of the CNTs' length. The obtained flexural strength behavior at these ages is nearly similar to the previous experiment.

On the 28th day, the strength results showed that CNTs with longer lengths and higher aspect ratios achieved slightly higher flexural strengths up to a CNTs-to-cement weight fraction of $0.15 \%$. The strength of the batches prepared at $0.03,0.08$, and $0.15 \%$ long CNTs of $10-30 \mu \mathrm{m}$ length increased by only 4,13 , and $3 \%$, respectively. However, for batches prepared at $0.25 \%$ short CNTs of $0.5-2 \mu \mathrm{m}$ length, higher flexural strength was obtained compared to similar batches with long CNTs by about 5\%. This may be related to the better dispersion capabilities of the short CNTs at high weight fractions. Compared to plain cement, the results showed that batches prepared at 0.15 and $0.25 \%$ CNTs concentrations resulted in higher flexural strengths by about $20 \%$.

Figure 6 shows the compressive strength results of CNTs' length test batches including the low standard deviations in most batches. The results showed that CNTs' lengths slightly influenced the compressive strength. On the 3rd and 7th days, the compressive strengths of the batches containing low CNTs' content of 0.03 and $0.08 \%$ were relatively lower than 

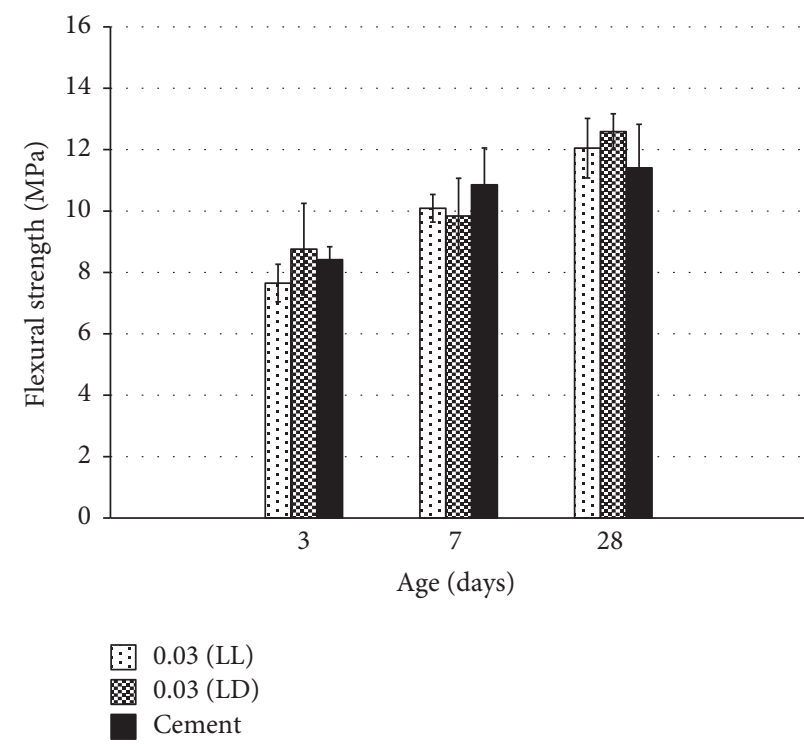

$\square$ Cement

(a)

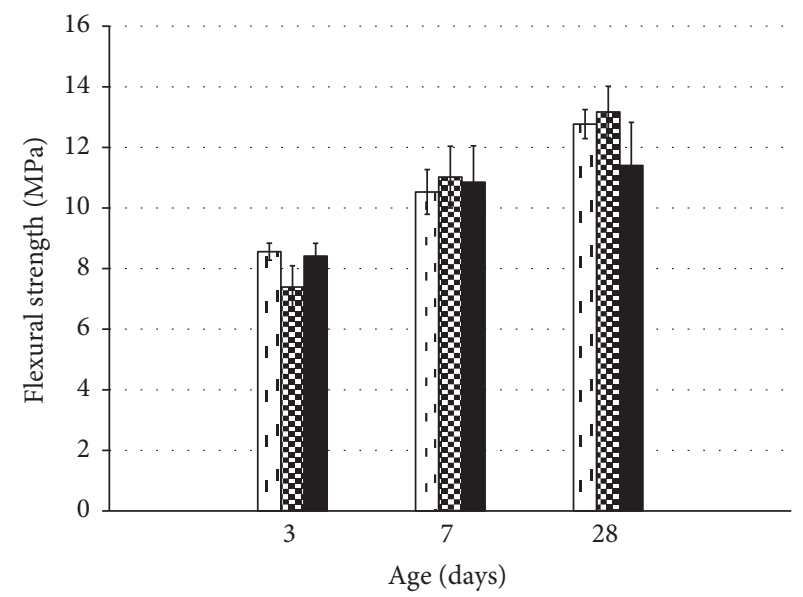

$0.15(\mathrm{LL})$

$0.15(\mathrm{LD})$

Cement

(c)

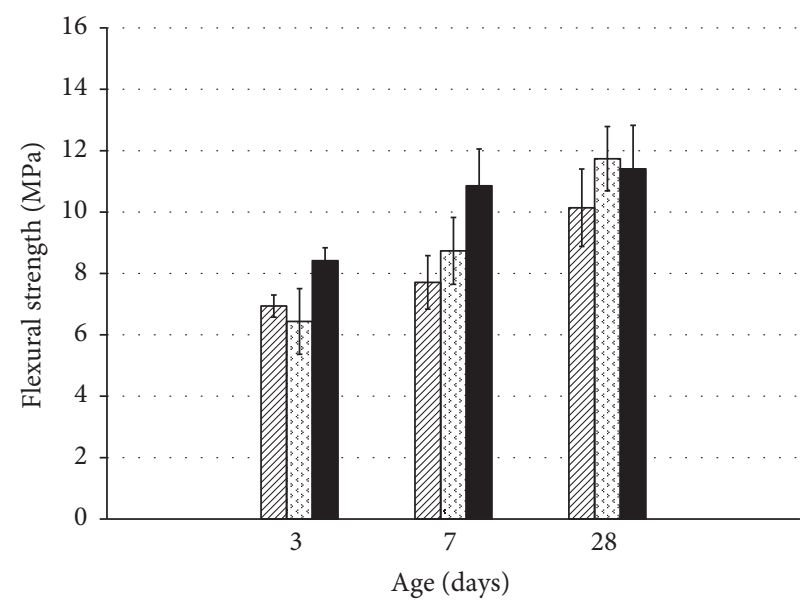

$0.08(\mathrm{LL})$
$0.08(\mathrm{LD})$
Cement

(b)

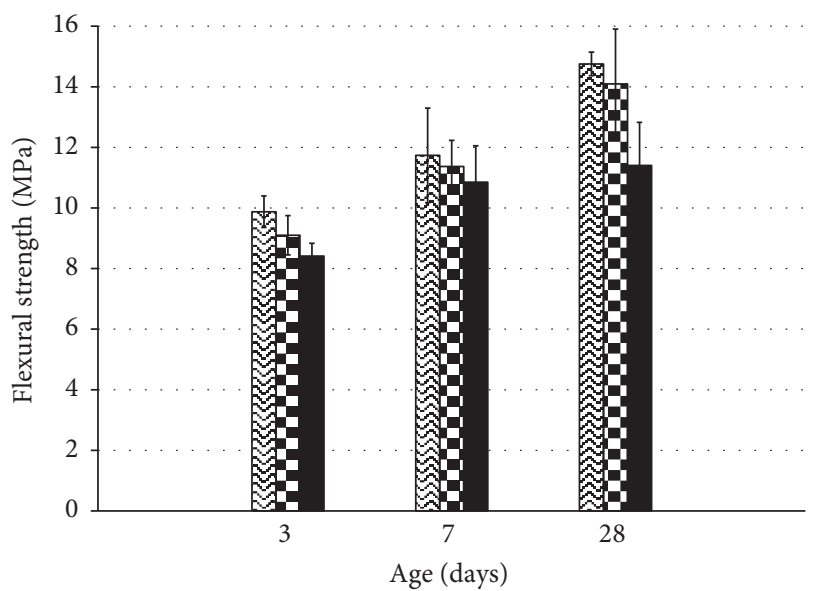

B $0.25(\mathrm{LL})$

$0.25(\mathrm{LD})$

Cement

Figure 5: CNTs' length test flexural strength results. (a) $0.03 \%$, (b) $0.08 \%$, (c) $0.15 \%$, and (d) $0.25 \%$ CNTs.

plain cement batch strength, whereas the strengths of the batches containing higher CNTs' content of 0.15 and $0.25 \%$ were higher than or comparable to the plain cement batch's strength regardless of the CNTs' length. On the 28th day and for batches prepared at $0.03 \%$ CNTs-to-cement weight fractions, the compressive strengths were similar to those of the plain cement batch. However, at batches prepared using $0.08 \%$ CNTs, the strength data were relatively lower compared to plain cement batches. For batches prepared using $0.15 \%$ CNTs, the compressive strengths of long CNTs were higher by $10 \%$ compared to plain cement. However, short CNTs of similar weight fraction had the same strength results as plain cement batches. For batches prepared using $0.25 \%$ CNTs, the compressive strengths of both CNTs lengths of $10-30 \mu \mathrm{m}$ and $0.5-2 \mu \mathrm{m}$ were higher by about $12 \%$ than plain cement strength.

\subsection{SEM Microstructural Analysis}

3.2.1. Effect of CNTs' Diameter. The microstructure investigation showed several observations linked to the dispersion and hydration elements of the samples. Figures 7, 8, and 9 illustrate the microstructure of batches containing 0.03 and $0.25 \%$ CNTs of high, medium, and low diameters of 20-30 and $10-20 \mathrm{~nm}$, respectively. Even though the samples with the highest aspect ratios provide the largest contact area between fibers and cement matrix, the images taken could not illustrate this information qualitatively. In terms of CNTs' weight 


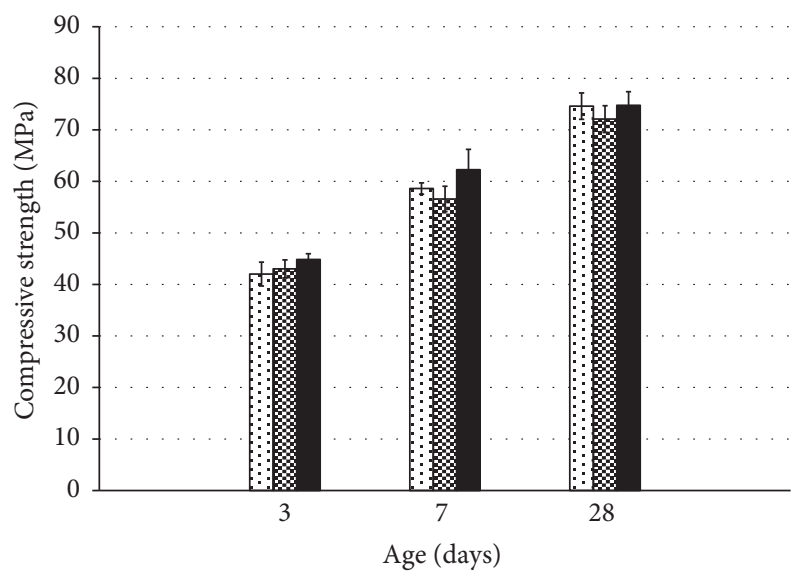

$0.03(\mathrm{LL})$
$0.03(\mathrm{LD})$
Cement

(a)

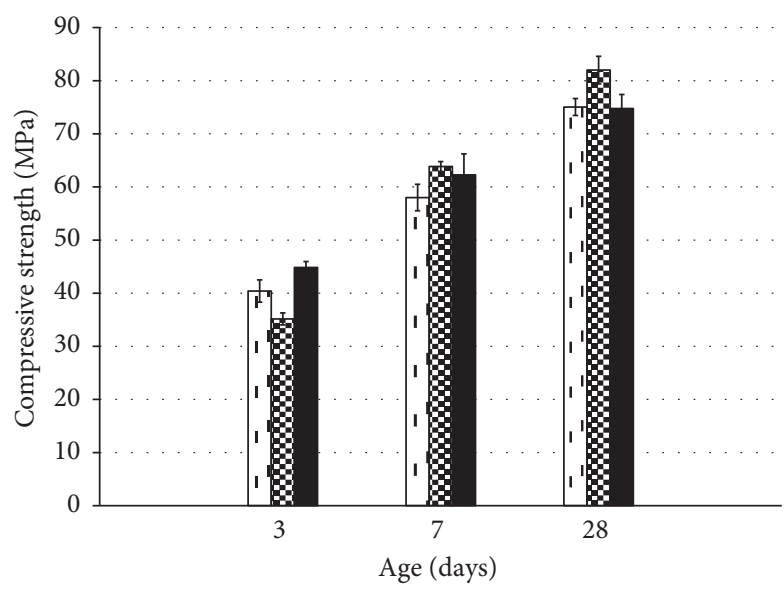

$0.15(\mathrm{LL})$
$0.15(\mathrm{LD})$
Cement

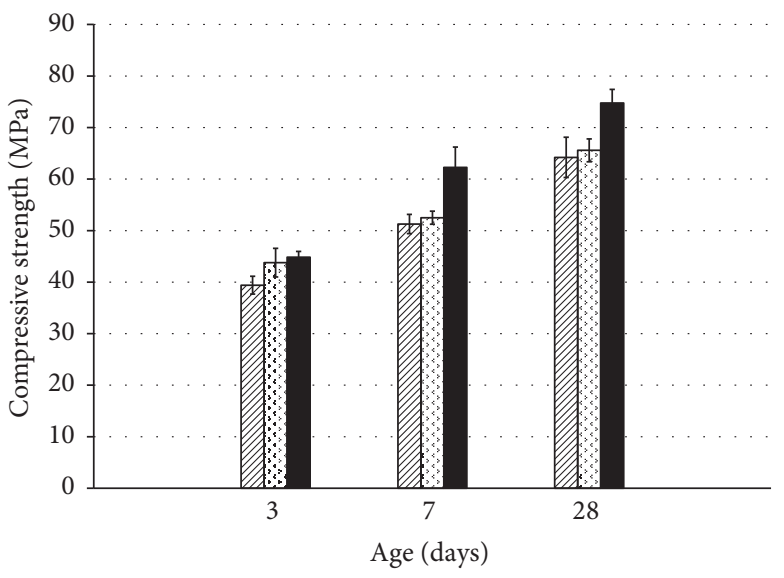

$0.08(\mathrm{LL})$

$0.08(\mathrm{LD})$

Cement

(b)

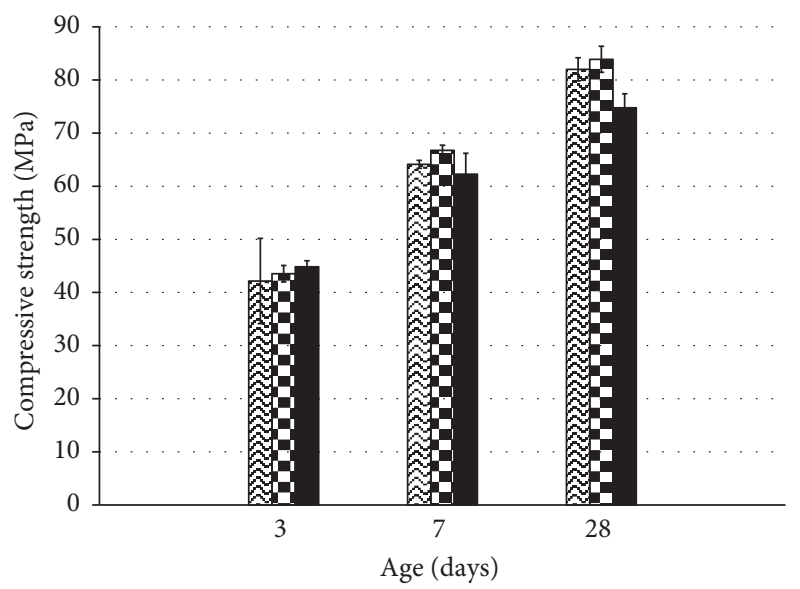

$0.25(\mathrm{LL})$
$0.25(\mathrm{LD})$

(c)

(d)

FigURE 6: CNTs' length test compressive strength results. (a) $0.03 \%$, (b) $0.08 \%$, (c) $0.15 \%$, and (d) $0.25 \%$ CNTs.

fraction, the microstructure investigation demonstrated that batches of $0.03 \%$ CNTs' content have better dispersion properties compared to batches containing higher CNTs' content of $0.25 \%$. This is related to the lower weight fraction of the nanofilaments which allows the tubes to be easily spread through the sonication process. The microstructural analysis also showed agglomerations of CNTs found at a few locations within the cement matrix in batches mixed with 0.15 and $0.25 \%$ CNTs of all diameters (Figures 7(b), 8(b), and $9(\mathrm{~b}))$. These agglomerations indicate the need to provide additional measures to eliminate CNTs' agglomerations. To compare with the strength results, the existence of CNTs' agglomerations in these batches did not affect their flexural and compressive strengths, which were higher than those of lower CNTs' contents of 0.03 and $0.08 \%$, as well as the plain cement batches.
3.2.2. Effect of CNTs' Length. Figures 10 and 11 show the microstructure of samples containing 0.03 and $0.25 \%$ short and long CNTs of $0.5-2$ and $10-30 \mu \mathrm{m}$, respectively. At a lower CNTs' content of $0.03 \%$, the images illustrated good dispersion properties for both short and long CNTs. However, at a high CNTs' content of $0.25 \%$, the images illustrated a better dispersion quality for batches of short CNTs. This could explain the increase in the flexural strength of these short CNTs' batches compared to the ones containing long CNTs of $0.25 \%$.

The microstructural analysis indicated that CNTs can be impeded or covered by cement hydration products. This finding was clearly shown for batches of short CNTs (Figure 12). Furthermore, it was observed that agglomerations of CNTs were also found at a few locations within the cement matrix regardless of the CNTs' length (Figure 13). It can be concluded 


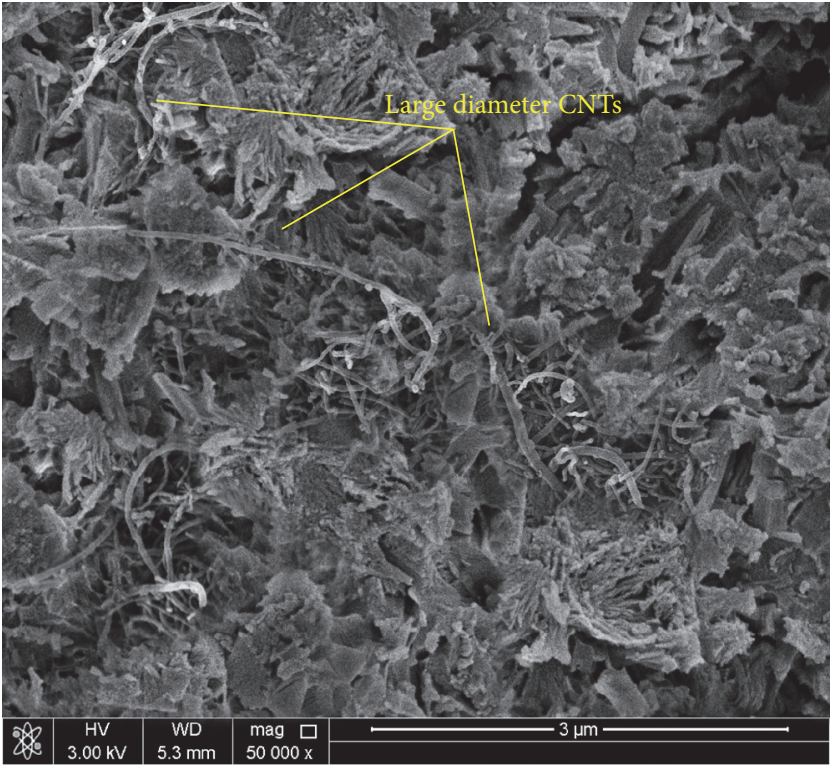

(a)

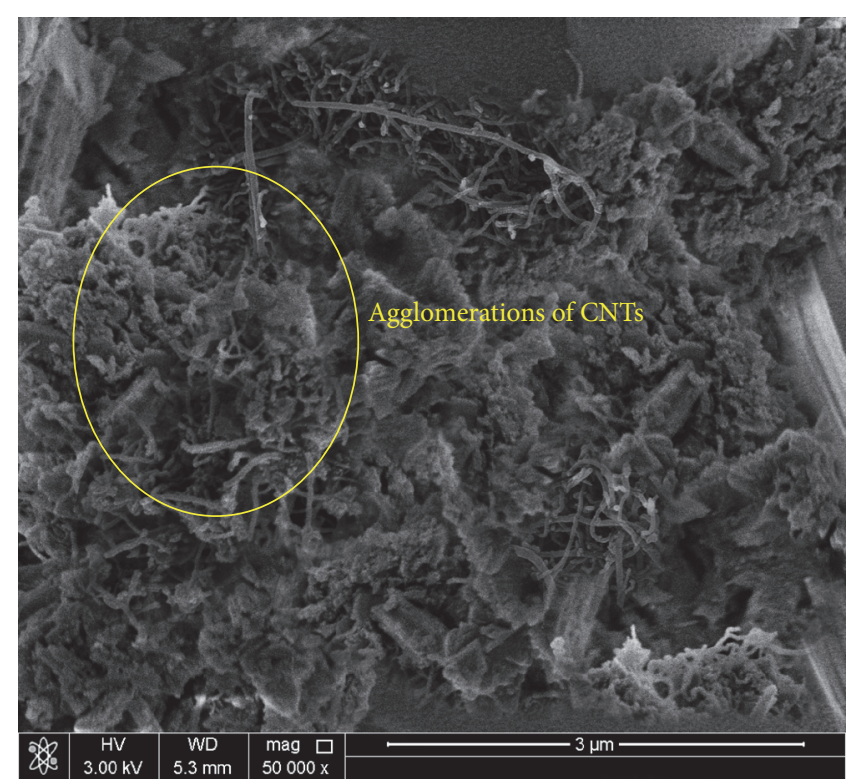

(b)

FIGURE 7: SEM images of large CNTs' diameters of 30-50 nm. (a) 0.03\% CNTs and (b) $0.25 \%$ CNTs.

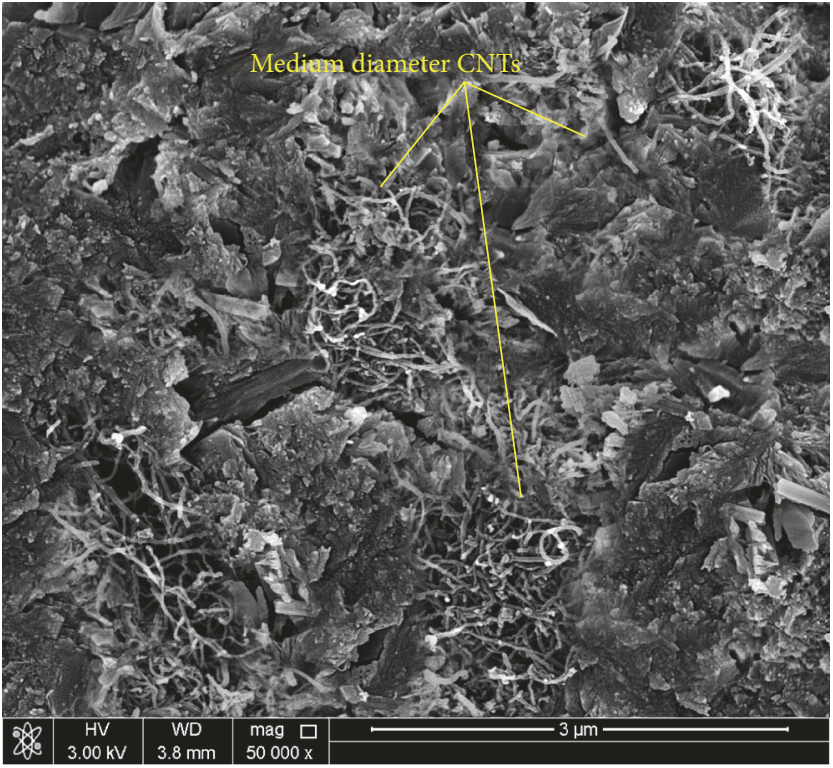

(a)

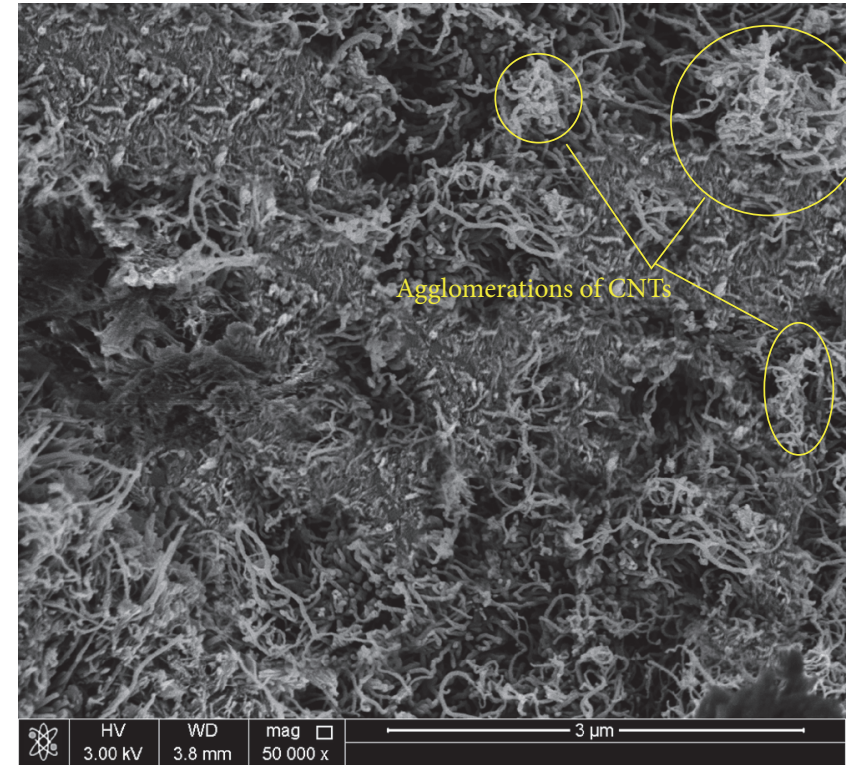

(b)

FIGURE 8: SEM images of medium CNTs' diameters of $20-30 \mathrm{~nm}$. (a) $0.03 \%$ CNTs and (b) $0.25 \%$ CNTs.

from the microstructural analysis that the weight fraction of the nanofilaments is the major factor affecting the overall composite strength rather than the length or diameter of the nanofilaments. Even though batches of lower CNTs' content of 0.03 and $0.08 \%$ showed better dispersion quality at many locations, their strength remained even lower than plain cement batches. On the other hand, batches of higher CNTs' content of 0.15 and $0.25 \%$ have always attained higher strength results despite the existence of CNTs' agglomerations in many spots.
3.3. RSM Analysis. The flexural strength prediction equation was determined as follows:

$$
\begin{aligned}
\mathrm{FS}= & 11.06+0.244 x_{1}+1.02 x_{2}-0.618 x_{1} x_{2}+1.28 x_{1}{ }^{2} \\
& +0.699 x_{2}{ }^{2}
\end{aligned}
$$

The $R^{2}$ and $P$ values of the flexural strength predicted model were determined as 0.76 and 0.0062 , respectively. 


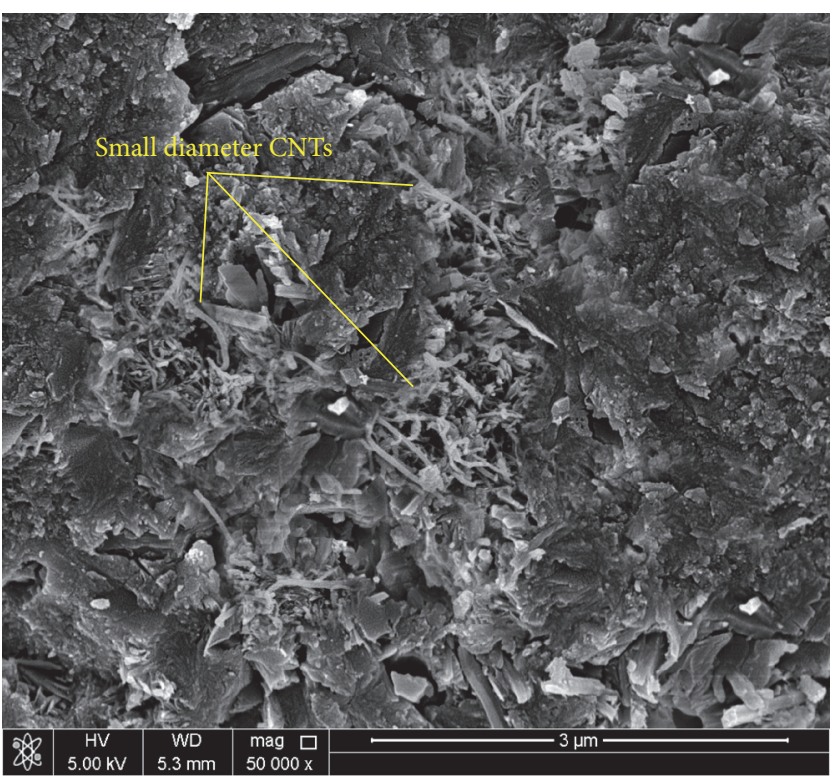

(a)

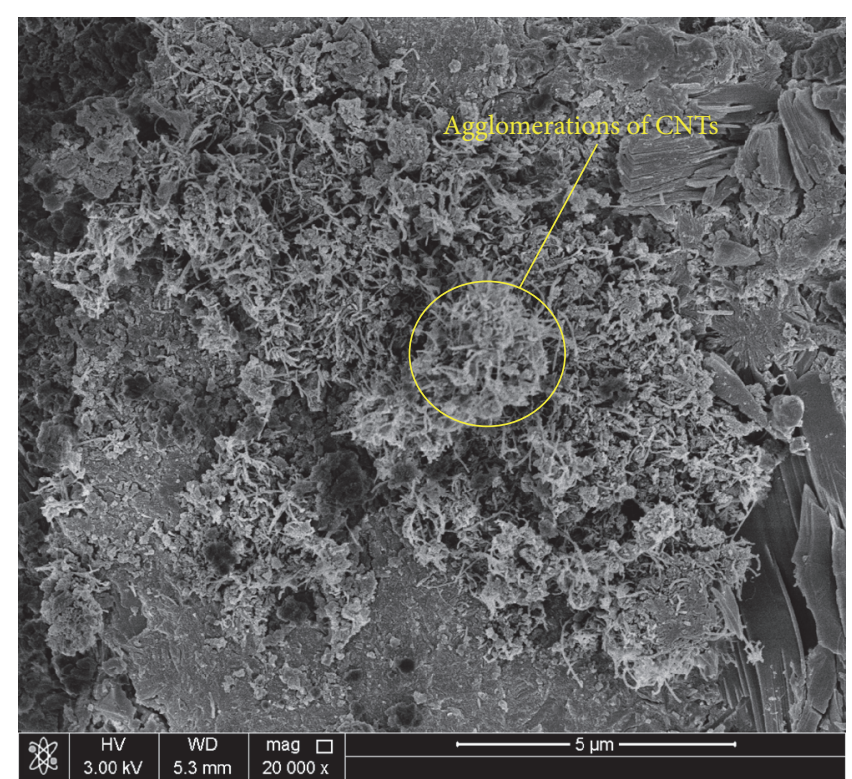

(b)

Figure 9: SEM images of small CNTs' diameters of $10-20 \mathrm{~nm}$. (a) $0.03 \%$ CNTs and (b) $0.25 \%$ CNTs.

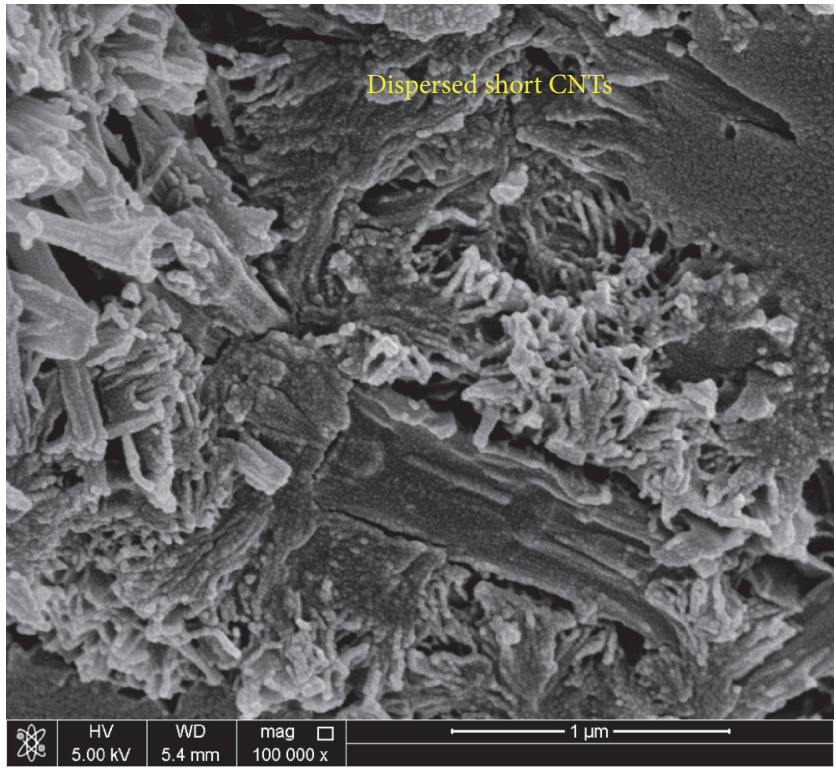

(a)

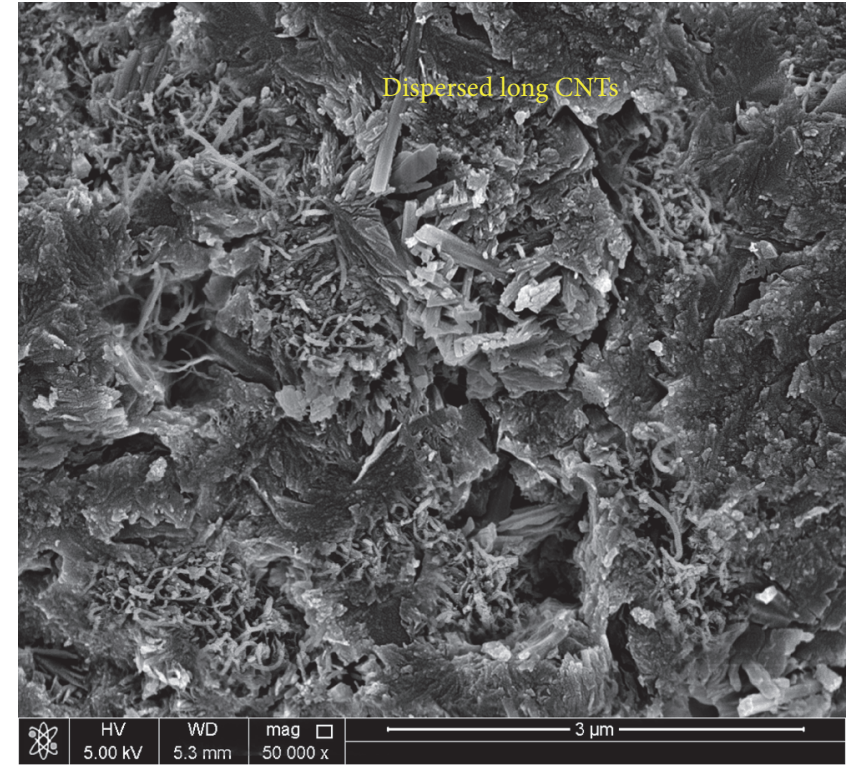

(b)

FIGURE 10: SEM images of $0.03 \%$ CNT samples. (a) Short CNTs (0.5-2 $\mu \mathrm{m}$ length) and (b) long CNTs (10-30 $\mu \mathrm{m}$ length).

On the other hand, the compressive strength prediction equation was determined as follows:

$$
\begin{aligned}
\mathrm{CS}= & 73.73+1.16 x_{1}+7.19 x_{2}+0.688 x_{1} x_{2}+0.45 x_{1}{ }^{2} \\
& +2.72 x_{2}{ }^{2} .
\end{aligned}
$$

The $R^{2}$ and $P$ values of the compressive strength predicted model were determined as 0.62 and 0.0512 , respectively.
The response surface plots of both flexural and compressive strength factors against the aspect ratio and weight fraction variables are shown in Figure 14. The flexural strength model (Figure 14(a)) shows peaks at all boundaries, except at the point of minimum aspect-weight fraction boundary. This indicates the effectiveness of CNTs in improving the flexural strength of cement paste with both minimum and maximum weight fractions of 0.03 and $0.25 \%$, respectively. On the other hand, the compressive strength model (Figure 14(b)) shows the peaks at both largest and smallest aspect ratios of 83 and 


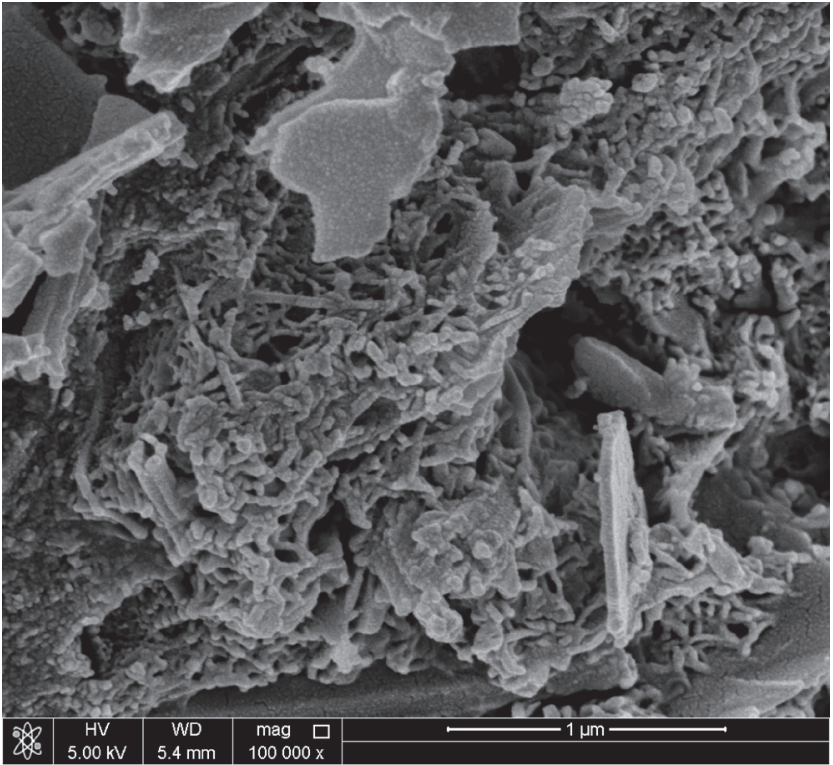

(a)

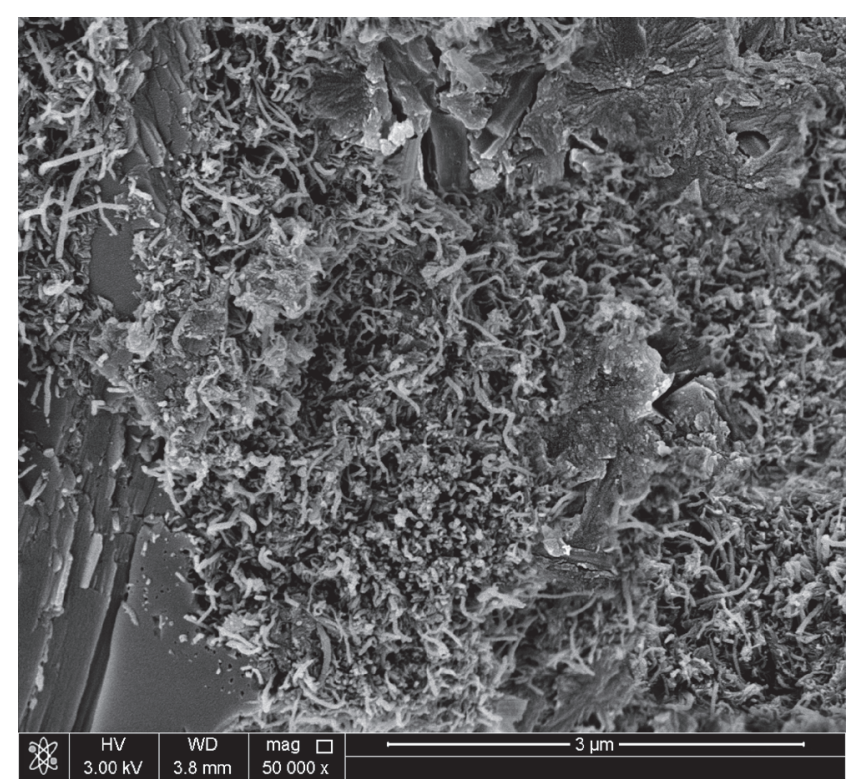

(b)

FIGURE 11: SEM images of $0.25 \%$ CNT samples. (a) Short CNTs $(0.5-2 \mu \mathrm{m}$ length) and (b) long CNTs (10-30 $\mu \mathrm{m}$ length).

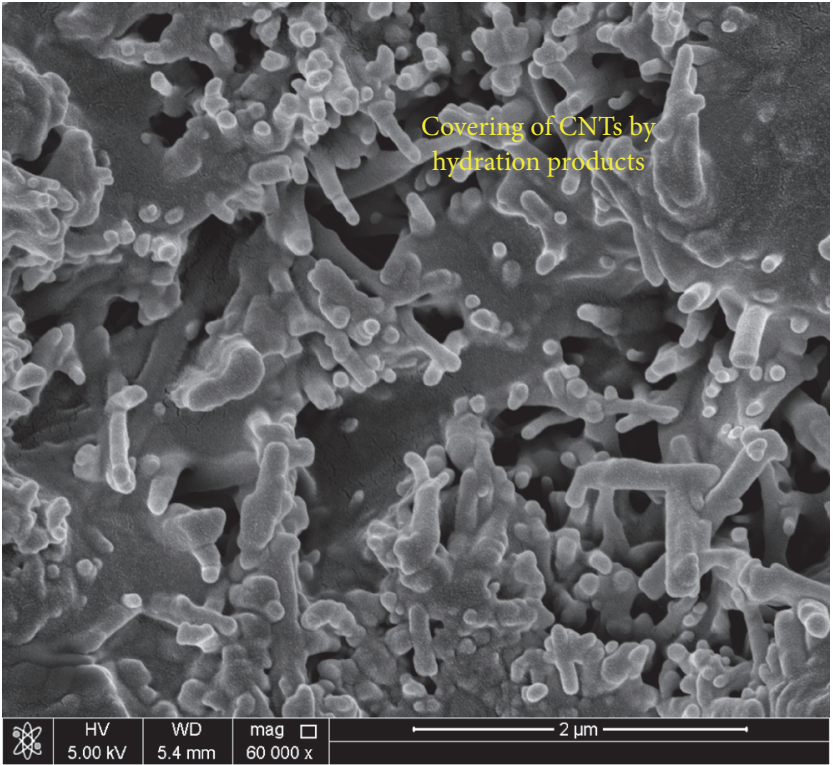

(a)

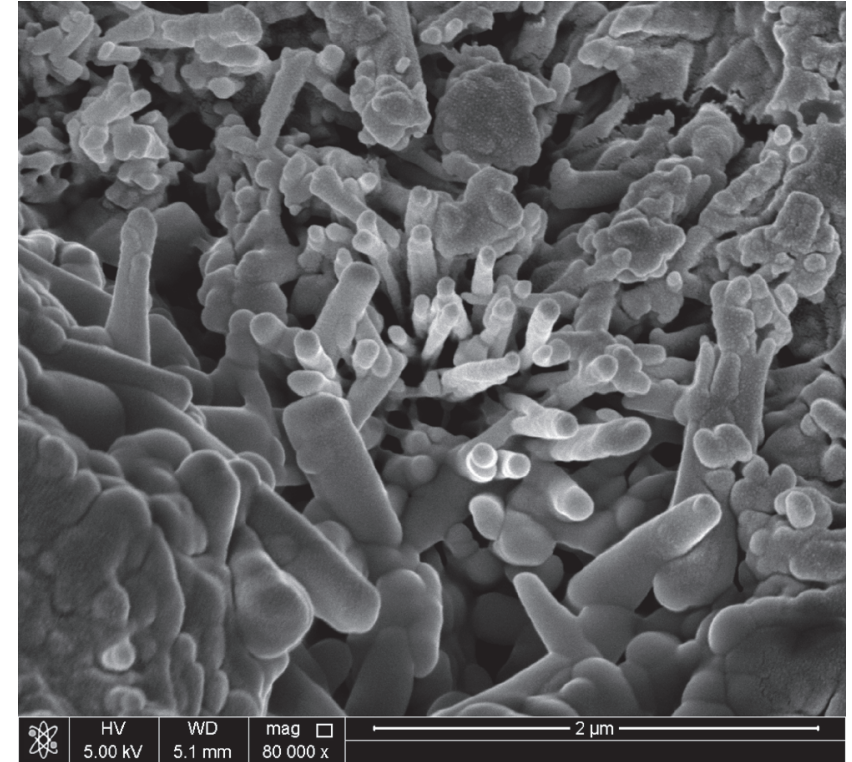

(b)

FIGURE 12: Short CNTs $(0.5-2 \mu \mathrm{m}$ length) impeded in cement hydration products. (a) Sample image 1 and (b) sample image 2.

1,333 of the highest weight fraction of $0.25 \%$, respectively. This response shows the importance of having CNTs' weight fraction as high as $0.25 \%$ to obtain the maximum compressive strength response.

Figure 15(a) shows the combined contour profiles of the aspect ratio and weight fraction variables versus the flexural and compressive strength. The shown shaded areas include all flexural and compressive strengths falling below the strengths of plain cement paste batch's flexural and compressive strengths. This analysis shows the importance of having a CNTs' weight fraction of more than $0.166 \%$, regardless of the aspect ratio or geometry in case an improvement of both flexural and compressive strengths of cement paste is required. Figure 15(b) shows the maximized desirability strength function behavior of both aspect ratio and weight fraction variables. The results showed that the maximum behavior would occur at the lowest aspect ratio of 83 at the highest weight fraction of $0.25 \%$. The maximum predicted flexural and compressive strengths are 14.4 and $82.3 \mathrm{MPa}$, respectively. Compared to the plain cement batch, these 


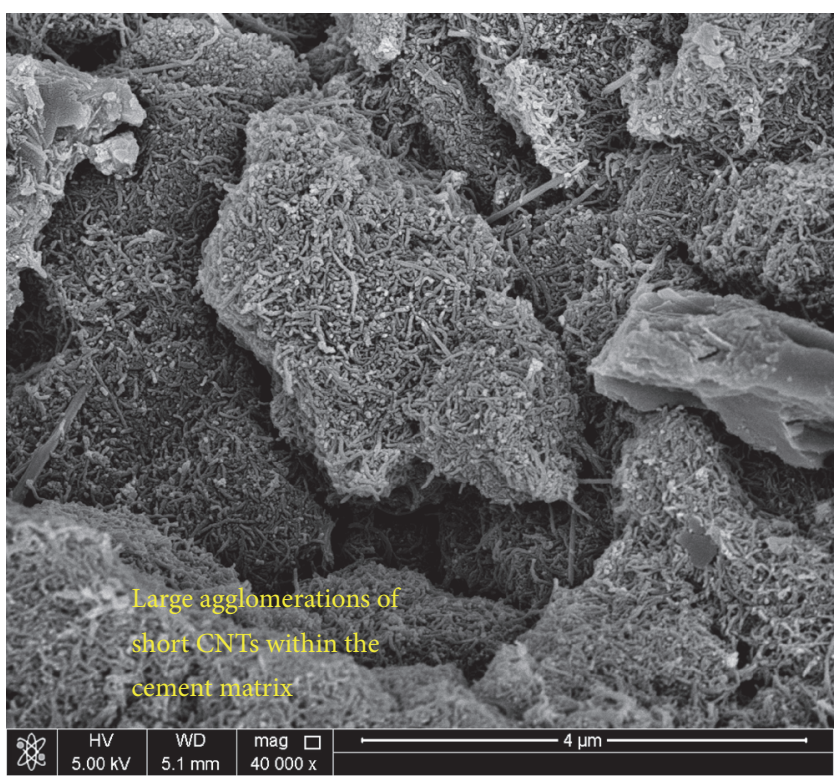

(a)

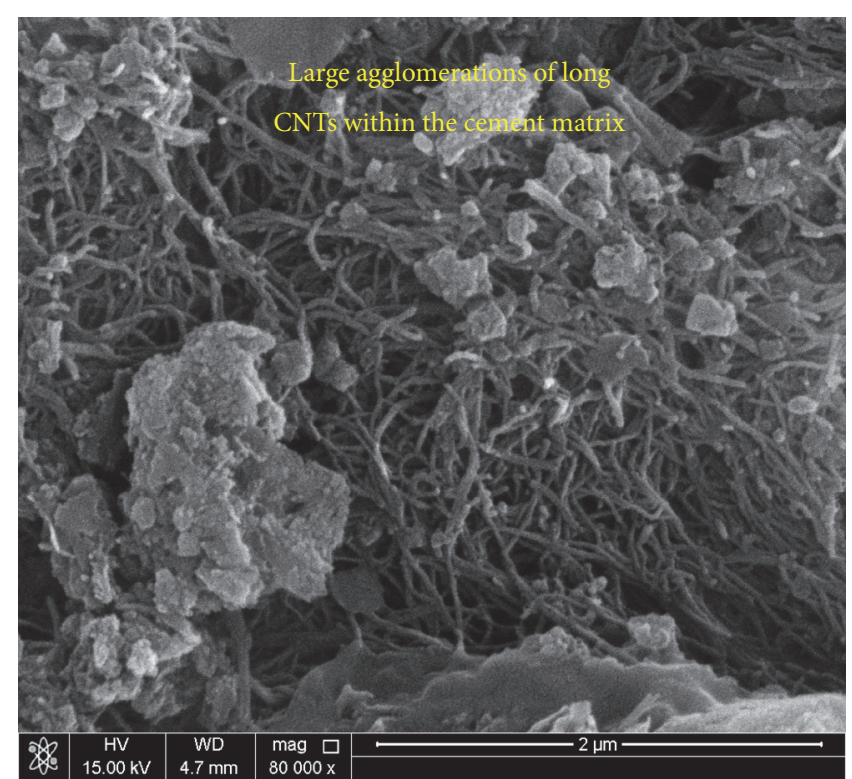

(b)

Figure 13: Agglomerations of CNTs within the cement matrix. (a) Batch with short CNTs (0.5-2 $\mu \mathrm{m}$ length) and (b) batch with long CNTs (10-30 $\mu \mathrm{m}$ length).

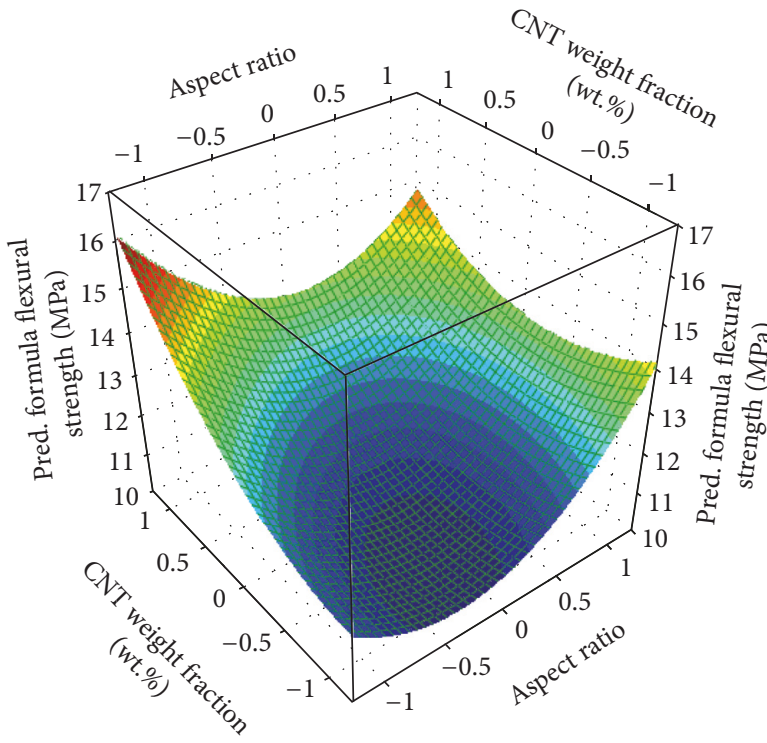

(a)

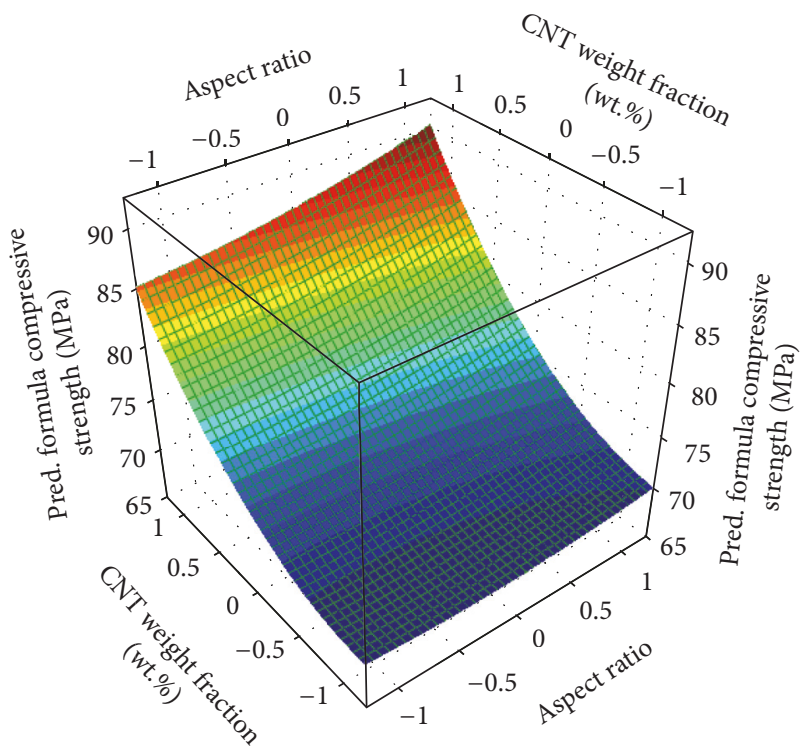

(b)

FIGURE 14: RSM models of strength factors against aspect ratio and weight fraction variables. (a) Flexural strength function's response and (b) compressive strength function's response.

values present an improvement of 26 and $10 \%$, respectively.

\section{Conclusions}

The effect of CNTs' geometry on the dispersion and flexural and compressive strengths of cement paste composites was investigated. The following conclusions are warranted at this time: (i) CNTs' diameter has a relatively slight effect on both flexural and compressive strengths' results.

(ii) CNTs of the lowest diameter of $10-20 \mathrm{~nm}$ and highest aspect ratio of 1,333 achieved higher strength results than CNTs of medium and high (20-30 and 30-50 nm) diameters, respectively.

(iii) CNTs with longer lengths of $10-30 \mu \mathrm{m}$ and higher aspect ratios of 1,333 achieved slightly higher flexural 


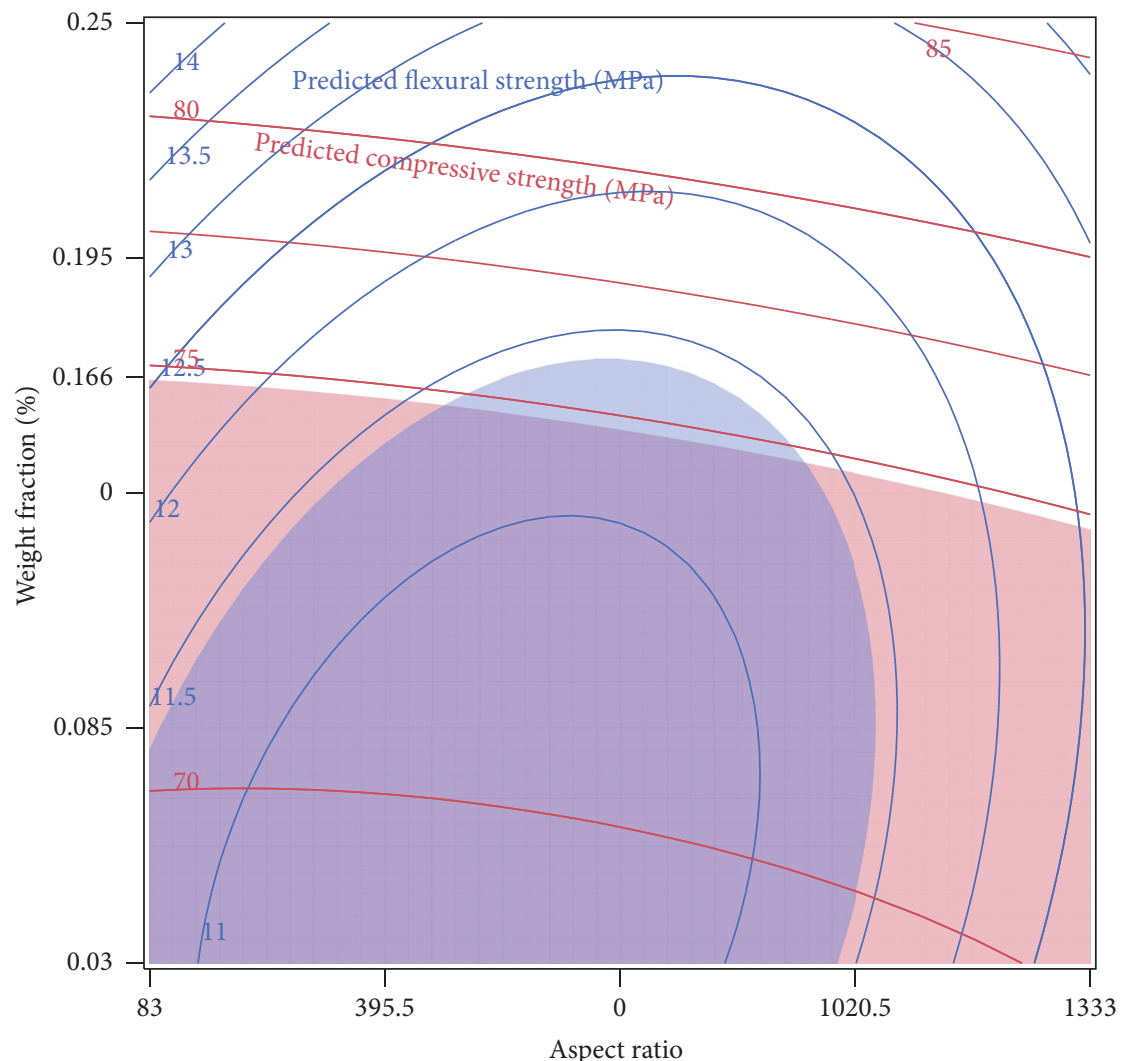

(a)

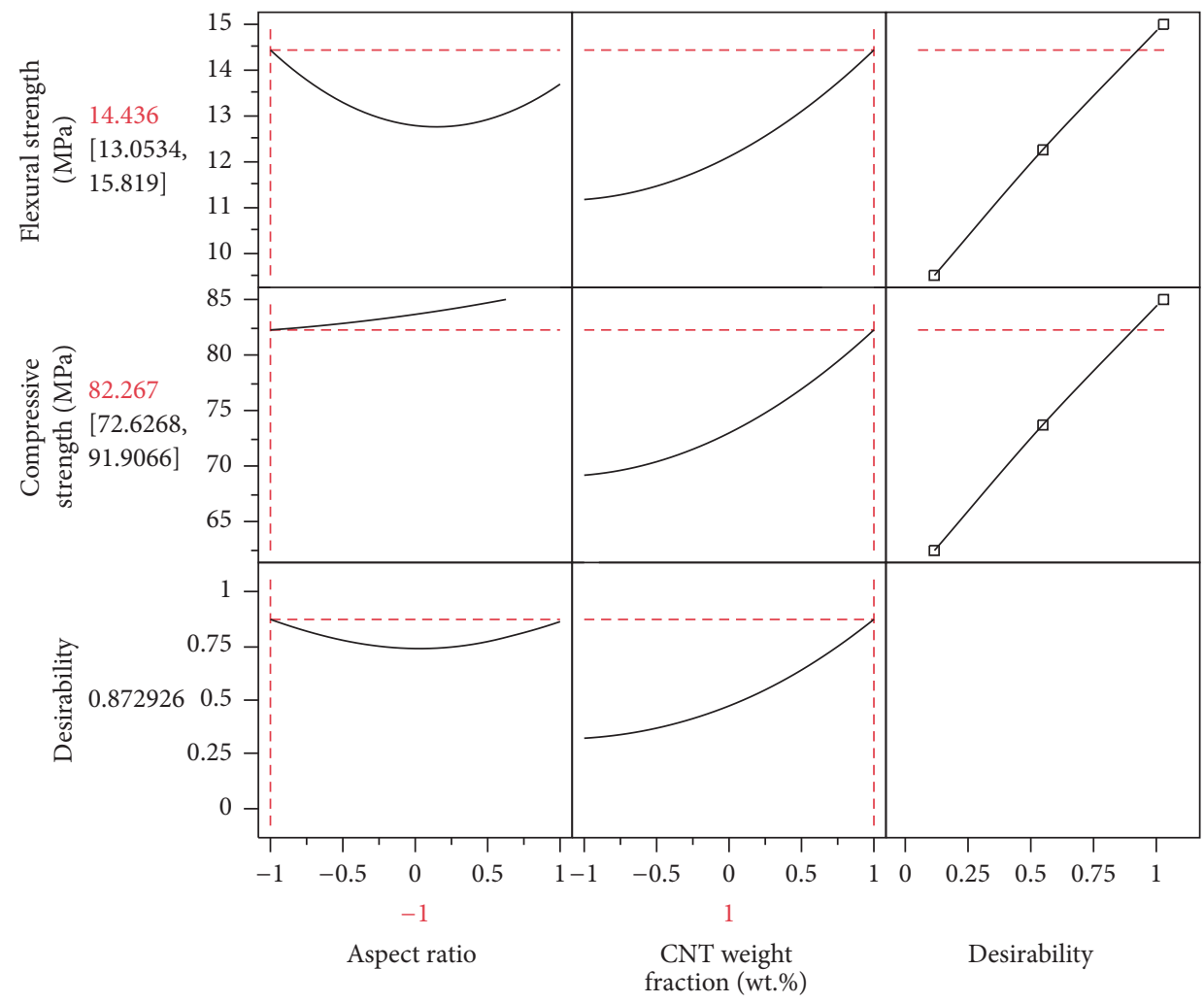

(b)

FIGURE 15: Aspect ratio and weight fraction variables. (a) Contour profiles and (b) maximized desirability functions. 
strengths using a CNTs'-to-cement weight fraction up to $0.15 \%$.

(iv) At a $0.25 \%$ CNTs content, short CNTs of $0.5-2 \mu \mathrm{m}$ length achieved higher flexural strengths than similar batches with long CNTs of 10-30 $\mu \mathrm{m}$ length.

(v) Compared to plain cement, samples reinforced with 0.15 and $0.25 \%$ CNTs attained higher strengths in both flexure and compression testing.

(vi) The microstructural qualitative analysis illustrated that better dispersion properties could be observed at lower CNTs' contents, regardless of the CNTs' geometry.

(vii) SEM analysis indicated that short CNTs $(0.5-2 \mu \mathrm{m}$ length) have better dispersion properties compared to long ones (10-30 $\mu \mathrm{m}$ length) at high CNTs' weight fraction.

(viii) RSM showed that maximum flexural and compressive strength responses were obtained at the lowest aspect ratio of 83 at the highest weight fraction of $0.25 \%$.

\section{Future Work}

This study aimed at investigating the effect of MWCNTs' geometry on the strength properties of the nanocementitious composites. Future work will be directed towards investigating the load-deflection curves to study such effect on other properties such as ductility, elasticity, and toughness.

\section{Conflicts of Interest}

The authors declare that they have no conflicts of interest.

\section{Acknowledgments}

The authors thank Qatar National Research Fund for their financial support (Grant no. NPRP 4-1142-2-440). Also, the authors are grateful to Mr. Essam Shabaan from the Central Laboratory Unit and Dr. Ahmed Al Khatat from the Department of Chemical Engineering at Qatar University for their support with the SEM and chemical analyses.

\section{References}

[1] M. M. J. Treacy, T. W. Ebbesen, and J. M. Gibson, "Exceptionally high Young's modulus observed for individual carbon nanotubes," Nature, vol. 381, no. 6584, pp. 678-680, 1996.

[2] A. Krishnan, E. Dujardin, T. W. Ebbesen, P. N. Yianilos, and M. M. J. Treacy, "Young's modulus of single-walled nanotubes," Physical Review B, vol. 58, no. 20, pp. 14013-14019, 1998.

[3] M.-F. Yu, O. Lourie, M. J. Dyer, K. Moloni, T. F. Kelly, and R. S. Ruoff, "Strength and breaking mechanism of multiwalled carbon nanotubes under tensile load," Science, vol. 287, no. 5453, pp. 637-640, 2000.

[4] E. W. Wong, P. E. Sheehan, and C. M. Lieber, "Nanobeam mechanics: elasticity, strength, and toughness of nanorods and nanotubes," Science, vol. 277, no. 5334, pp. 1971-1975, 1997.
[5] K. Holschemacher and Y. Klug, "A database for the evaluation of hardened properties of SCC," LACER, vol. 7, pp. 123-134, 2002.

[6] S. Musso, J.-M. Tulliani, G. Ferro, and A. Tagliaferro, "Influence of carbon nanotubes structure on the mechanical behavior of cement composites," Composites Science and Technology, vol. 69, no. 11-12, pp. 1985-1990, 2009.

[7] A. Yazdanbakhsh, Z. C. Grasley, B. Tyson, and R. K. Abu AlRub, "Carbon nano filaments in cementitious materials: some issues on dispersion and interfacial bond," ACI Special Publication, vol. 267, pp. 21-34, 2009.

[8] R. K. Abu Al-Rub, A. I. Ashour, and B. M. Tyson, "On the aspect ratio effect of multi-walled carbon nanotube reinforcements on the mechanical properties of cementitious nanocomposites," Construction and Building Materials, vol. 35, pp. 647-655, 2012.

[9] A. Sobolkina, V. Mechtcherine, V. Khavrus et al., "Dispersion of carbon nanotubes and its influence on the mechanical properties of the cement matrix," Cement and Concrete Composites, vol. 34, no. 10, pp. 1104-1113, 2012.

[10] J. N. Paula, J. M. Calixto, L. O. Ladeira et al., "Mechanical and rheological behavior of oil-well cement slurries produced with clinker containing carbon nanotubes," Journal of Petroleum Science and Engineering, vol. 122, pp. 274-279, 2014.

[11] R. Siddique and A. Mehta, "Effect of carbon nanotubes on properties of cement mortars," Construction and Building Materials, vol. 50, pp. 116-129, 2014.

[12] A. G. Abu Taqa, R. K. Abu Al-Rub, A. Senouci, N. Al-Nuaimi, and K. B. Hani, "The effect of fiber geometry and interfacial properties on the elastic properties of cementitious nanocomposite material," Journal of Nanomaterials, vol. 2015, Article ID 283579, 14 pages, 2015.

[13] S. Xu, J. Liu, and Q. Li, "Mechanical properties and microstructure of multi-walled carbon nanotube-reinforced cement paste," Construction and Building Materials, vol. 76, pp. 16-23, 2015.

[14] B. Zou, S. J. Chen, A. H. Korayem, F. Collins, C. M. Wang, and W. H. Duan, "Effect of ultrasonication energy on engineering properties of carbon nanotube reinforced cement pastes," Carbon, vol. 85, pp. 212-220, 2015.

[15] M. O. Mohsen, N. Al-Nuaimi, R. K. Abu Al-Rub, A. Senouci, and K. A. Bani-Hani, "Effect of mixing duration on flexural strength of multi walled carbon nanotubes cementitious composites," Construction and Building Materials, vol. 126, pp. 586598, 2016.

[16] S. Chuah, Z. Pan, J. G. Sanjayan, C. M. Wang, and W. H. Duan, "Nano reinforced cement and concrete composites and new perspective from graphene oxide," Construction and Building Materials, vol. 73, pp. 113-124, 2014.

[17] Y. Hu, D. Luo, P. Li, Q. Li, and G. Sun, "Fracture toughness enhancement of cement paste with multi-walled carbon nanotubes," Construction and Building Materials, vol. 70, pp. 332338, 2014.

[18] A. Al-Dahawi, O. Öztürk, F. Emami, G. Yildirim, and M. Şahmaran, "Effect of mixing methods on the electrical properties of cementitious composites incorporating different carbonbased materials," Construction and Building Materials, vol. 104, pp. 160-168, 2016.

[19] B. Wang, Y. Han, and S. Liu, "Effect of highly dispersed carbon nanotubes on the flexural toughness of cement-based composites," Construction and Building Materials, vol. 46, pp. 8-12, 2013.

[20] X. Wang, P. Bradford, Q. Li, and Y. Zhu, Aligned Carbon Nanotube Composite Prepregs, Nanotube Superfiber Materials, William Andrew Publishing, Waltham, MA, USA, 2014. 
[21] M. S. Konsta-Gdoutos, Z. S. Metaxa, and S. P. Shah, "Multiscale mechanical and fracture characteristics and early-age strain capacity of high performance carbon nanotube/cement nanocomposites," Cement and Concrete Composites, vol. 32, no. 2, pp. 110-115, 2010.

[22] B. M. Tyson, R. K. Abu Al-Rub, A. Yazdanbakhsh, and Z. Grasley, "Carbon nanotubes and carbon nanofibers for enhancing the mechanical properties of nanocomposite cementitious materials," Journal of Materials in Civil Engineering, vol. 23, no. 7, pp. 1028-1035, 2011. 

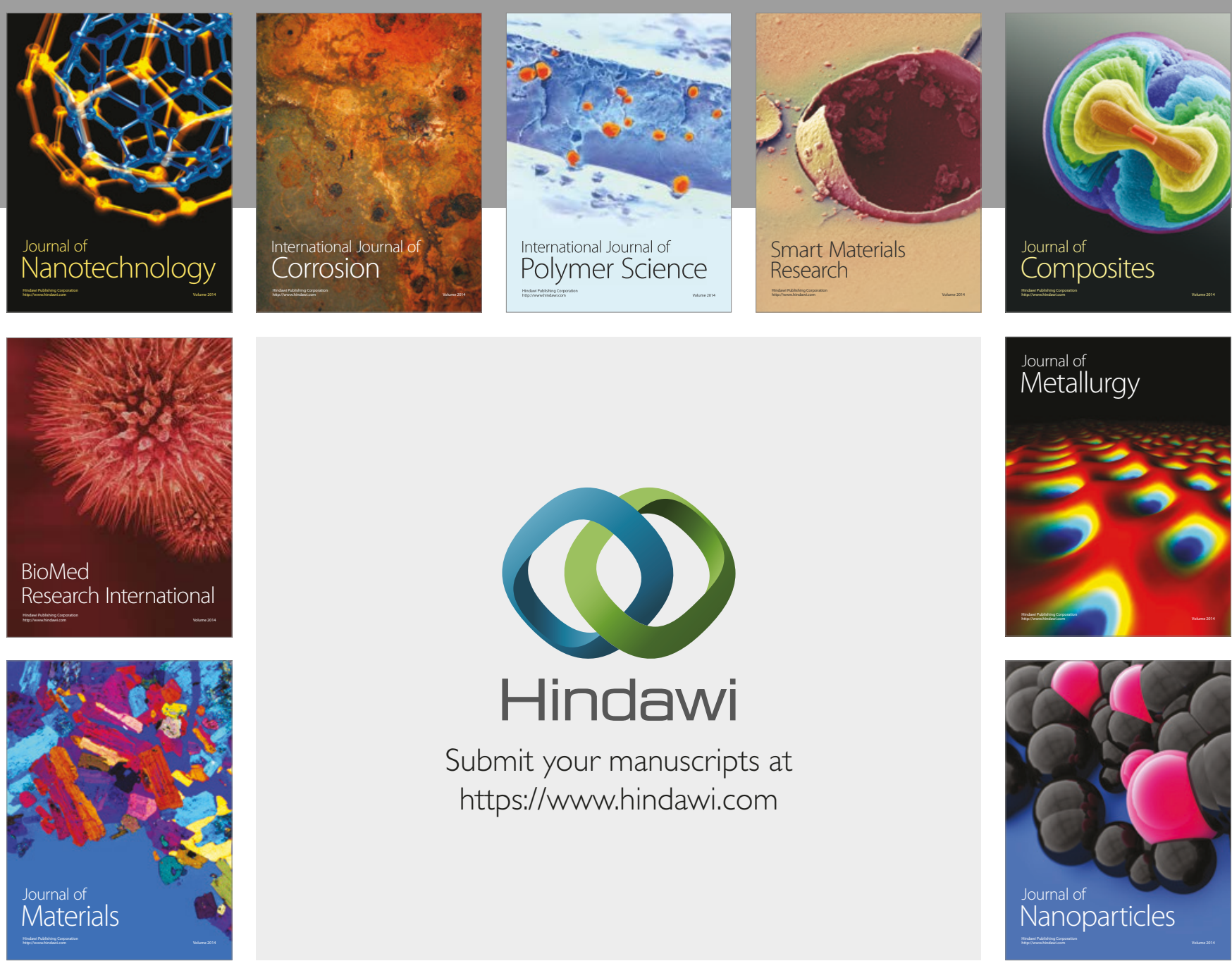

\section{Hindawi}

Submit your manuscripts at

https://www.hindawi.com
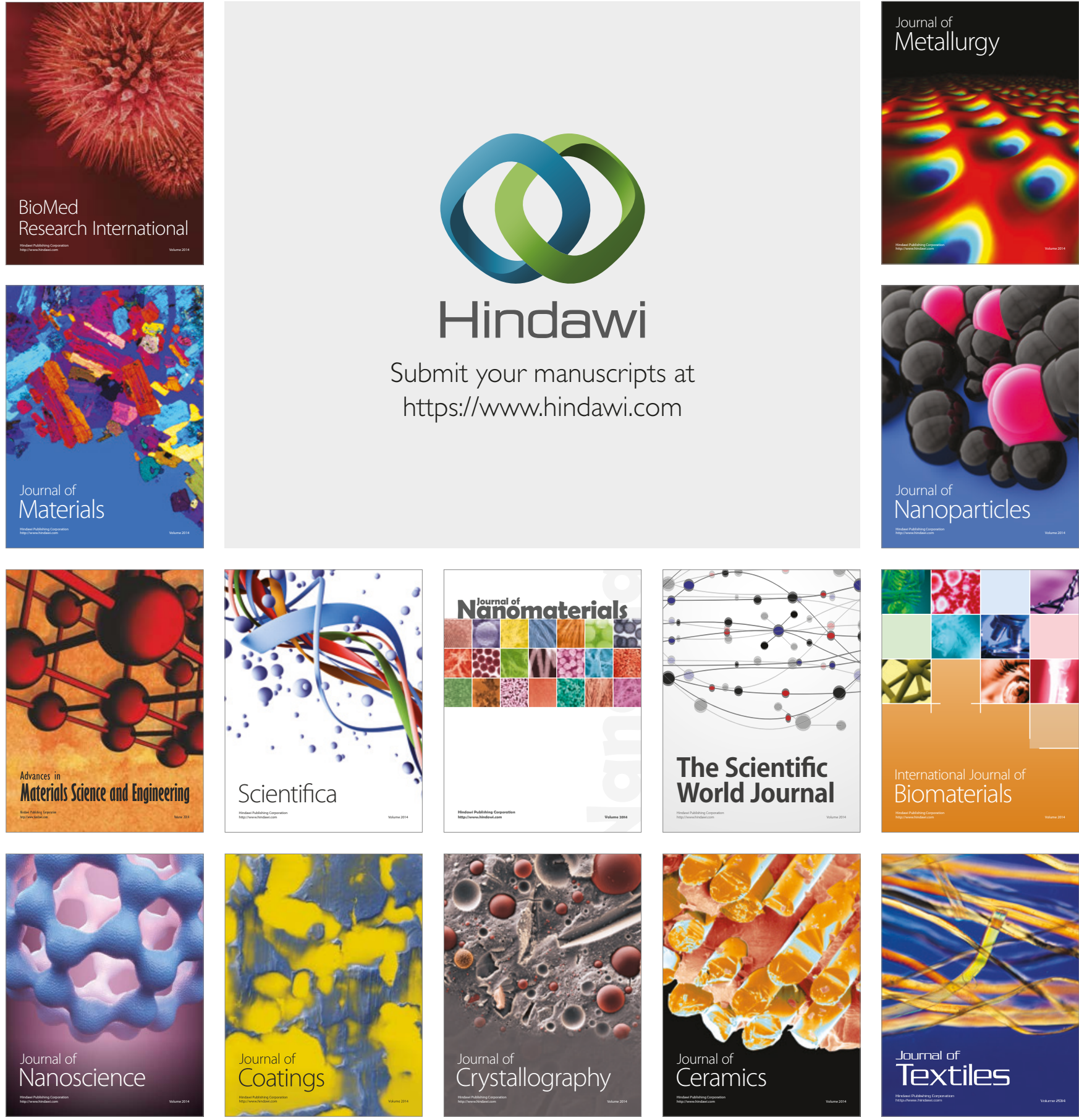

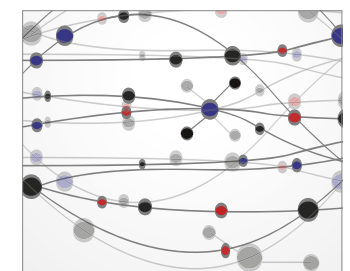

The Scientific World Journal
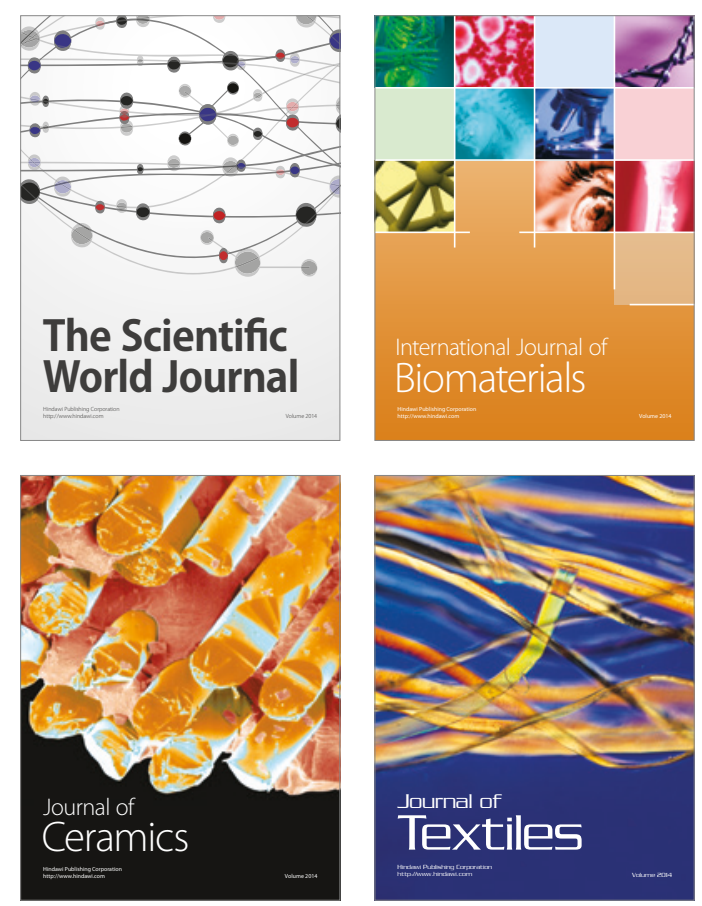